\title{
Soft tissue sarcomas with complex genomic profiles
}

\author{
Louis Guillou • Alain Aurias
}

Received: 12 July 2009 /Revised: 5 October 2009 / Accepted: 17 October 2009 /Published online: 13 November 2009

(C) Springer-Verlag 2009

\begin{abstract}
Soft tissue sarcomas (STS) with complex genomic profiles $(50 \%$ of all STS) are predominantly composed of spindle cell/pleomorphic sarcomas, including leiomyosarcoma, myxofibrosarcoma, pleomorphic liposarcoma, pleomorphic rhabdomyosarcoma, malignant peripheral nerve sheath tumor, angiosarcoma, extraskeletal osteosarcoma, and spindle cell/pleomorphic unclassified sarcoma (previously called spindle cell/pleomorphic malignant fibrous histiocytoma). These neoplasms show, characteristically, gains and losses of numerous chromosomes or chromosome regions, as well as amplifications. Many of them share recurrent aberrations (e.g., gain of 5p13-p15) that seem to play a significant role in tumor progression and/or metastatic dissemination. In this paper, we review the cytogenetic, molecular genetic, and clinicopathologic characteristics of the most common STS displaying complex genomic profiles. Features of diagnostic or prognostic relevance will be discussed when needed.
\end{abstract}

Keywords Soft tissue sarcoma $\cdot$ Karyotype $\cdot$ Genetics

\section{Guillou ( $\square)$}

University Institute of Pathology,

Centre Hospitalier Universitaire Vaudois and University of Lausanne,

Rue du Bugnon 25,

1011 Lausanne, Switzerland

e-mail: louis.guillou@chuv.ch

\section{A. Aurias}

Genetics and Biology of Cancers, and INSERM U830,

Institut Curie,

Paris, France

\section{Introduction}

Soft tissue sarcoma (STS) is a heterogenous group of mesenchymal neoplasms showing high variations in their clinical presentation, morphology, immunoprofile, genetics, and behavior. Roughly, STS can divided into several categories, namely those bearing reciprocal translocations accounting for $15-20 \%$ of cases (e.g., synovial sarcoma, Ewing sarcoma), those with specific mutations (e.g., gastrointestinal stromal tumor (GIST)) or deletions (e.g., rhabdoid tumor), those with amplifications (e.g., welldifferentiated liposarcoma), and those with complex genomic profiles (complex karyotypes). The latter category, which accounts for about $50 \%$ of STS, is mainly composed of spindle cell/pleomorphic tumors, including leiomyosarcoma, myxofibrosarcoma, pleomorphic liposarcoma (PLPS), pleomorphic rhabdomyosarcoma (PRMS), malignant peripheral nerve sheath tumor (MPNST), angiosarcoma, extraskeletal osteosarcoma, and spindle cell/ pleomorphic unclassified sarcoma (previously called spindle cell/pleomorphic malignant fibrous histiocytoma (MFH)). High-grade dedifferentiated liposarcoma which is also characterized by complex karyotype shows, in addition, distinctive genomic abnormalities (giant chromosomes, supernumerary ring chromosomes, amplification of 12q13-q21), paralleling that observed in well-differentiated liposarcoma (see chapter on well-differentiated and dedifferentiated liposarcomas).

\section{Leiomyosarcoma}

Leiomyosarcoma (LMS) accounts for about $8-10 \%$ of adult soft tissue sarcomas [1-3]. These malignant neoplasms which show varying degree of smooth muscle differentia- 
tion can develop anywhere in the body, as well as in visceral organs (prostate, urinary bladder, uterus, etc.).

\section{Cytogenetics and molecular genetics}

LMS usually show complex karyotypic alterations [4-6], and karyotypes differ generally from one tumor to another. Many LMS show chromosomal imbalances aberrations in the form of gains (chromosomes 1, 5, 6, 8, 15, 16, 17, 19, 20, 22, X), losses (chromosomes 1p, 2, 3, 4, 6q, 8, 9, 10p, $11 \mathrm{p}, 12 \mathrm{q}, 11 \mathrm{q}, 13,16,17 \mathrm{p}, 18$ 19, 22q), and amplifications (chromosomes 1, 5, 8, 12, 13, 17, 19, 20) [4, 6, 7].

Some gains and losses of chromosomal material, however, are more frequently observed and tend to correlate with poor outcome, large tumor size, and metastatic dissemination: loss of 1p12-pter, loss of $2 p$, loss of 13q14-q21 (targeting the Rb pathway) [8], loss of 10q (targeting PTEN) [9], and loss of 16q and gains of 17p, 8q, and $5 \mathrm{p} 14$ pter $[8,9]$. LMS often show loss of RB1, correlating with the high frequency of LMS observed in hereditary retinoblastoma patients. The 5p13-p15 region is often amplified in several pleomorphic STS including LMS. Residing in this amplicon are three candidates genes which are overexpressed: TRIO, NKD2, and IRX2, of which TRIO seems to be particularly involved in tumor progression of LMS [10]. Activation of the PI3K-AKT pathway through different mechanisms (e.g., activation of IGFR, inactivation of PTEN, a negative regulator of the PI3K-AKT) also plays a crucial role in the development and maintenance of LMS [11]. This activation leads to the concomitant activation of downstream effectors such as mTOR and its targets (ß-catenin, pS6, p4E-BP1, etc.), as well as to MDM2 stabilization [11]. Recent clinical trials showed that analogs of rapamycin such as everolimus (RAD001), an mTOR inhibitor, have some efficacy in patients with LMS and PEComas [12, 13]. Gene and protein expression studies also identified genes/proteins that seem to be associated with unfavorable prognosis and high metastatic risk in leiomyosarcomas (see also chapter of Beck et al.) [14-16], including mutations in TP53 gene and inactivation of $\mathrm{p} 16^{\mathrm{INK} 4 \mathrm{a}}[17,18]$, RASSF1A [19], and MGMT [20] secondary to promoter hypermethylation. A recent study on gene expression signature of 177 sarcomas identified a significant relationship between upegulation of several hypoxia-induced genes such as HIF1A and its targets and increased metastatic potential [21]. This was particularly true for STS with complex karyotypes, including LMS. This application of expression data would allow us to identify high-risk patients who might deserve specific therapy.

Recently, it has been realized that the more differentiated retroperitoneal leiomyosarcomas tend to behave more aggressively and that this aggressiveness was mainly dependent upon myocardin amplification/overexpression [22]. Myocardin is a transcriptional cofactor of SRF regulating smooth muscle differentiation. Pérot et al. [22] showed that inactivation of the myocardin pathway resulted in a significant reduction of smooth muscle differentiation, cell proliferation, and cell migration and was associated with less differentiated histology. These data suggest that myocardin might constitute a promising therapeutic target.

Some LMS which occur in children and some immunocompromised patients are associated with Epstein-Barr virus infection. EBV infection can be confirmed by in situ hybridization (using Epstein-Barr virus early RNA-EBER probes) or polymerase-chain-reaction-based methods [23], pointing to a potential role of EBV in leiomyosarcoma genesis. This was not observed in other neoplasms such as GIST [24].

Leiomyosarcomas of the uterus tend to parallel LMS of soft tissue in terms of karyotypic abnormalities. Most frequent losses by CGH include 10q, 11q, 13q, and $2 p$, whereas the most common gains are Xp, 1q, 5p, 8q, and $17 p[4,25]$. Aberrations involving $1 \mathrm{q} 21$ seem to be more common and aberrations of $1 \mathrm{p} 13$ and $10 \mathrm{q} 22$ less common in soft tissue leiomyosarcomas $[4,5]$. Losses involving 1q and $3 p$ are more frequent among soft tissue LMS whereas losses of chromosomes 14 and 15 and of 22q12-qter are more frequent in non-soft-tissue LMS [4]. TP53 mutations, MDM2 stabilization, and inactivation of $\mathrm{p} 16^{\mathrm{INK} 4 \mathrm{a}}$ are observed in both soft tissue and uterine LMS and seem to be associated with LMS progression. Leiomyoma and LMS of the uterine corpus are two different diseases, and there is no evidence that the former originates from the latter [26]. For example, TP53 mutations and loss of heterozygosity for chromosome 10 are not observed in leiomyomas, while the $\mathrm{t}(12 ; 14)$ ( $\mathrm{q} 15 ; \mathrm{q} 23-24)$ seen in many leiomyomas is not a feature of LMS $[4,25]$.

\section{Clinical features}

LMS are more common in middle-aged and elderly patients and rare in children. Clinical behavior depends mainly on tumor location $[1,2]$. Leiomyosarcomas of the dermis are mostly observed in lower extremities of middle-aged to older male individuals. They are often small $(<2 \mathrm{~cm})$ and have an excellent prognosis. LMS of the somatic soft tissues often present as large ( $6 \mathrm{~cm}$ on average) lesions which tend to be situated in the extremities, especially the thigh, frequently connected to a small vein (one third of cases). About half of them develop in the subcutis and the remainder in muscle. Metastases develop in $40 \%$ to $45 \%$ of patients with a 5-year survival rate of $64 \%$ [2, 27].

LMS of the retroperitoneum, abdominal cavity, and mediastinum account for $50-65 \%$ of all LMS. They occur preferentially in women (two third of cases), presenting as 
large (often $>10 \mathrm{~cm}$ ) multinodular masses which tend to infiltrate adjacent structures such as the kidney or pancreas. The prognosis of LMS of the abdominal cavity and retroperitoneum is markedly poor, with $70-90 \%$ of patients dying of their disease within 5 years $[2,27]$.

Epstein-Barr-virus-associated LMS tend to occur in children, to be multicentric, and to behave as low-grade tumors. They also occur in immunocompromised patients (e.g., transplanted patients and patients with acquired immunodeficiency syndrome) [23].

\section{Pathologic features}

Histologically, LMS is composed of a varying proportion of spindle and pleomorphic cells depending on the degree of differentiation. In well-differentiated lesions, the spindle cells are classically arranged in recognizable longitudinally oriented fascicles intersecting at right angles (Fig. 1). The cytoplasm of the cells is variably eosinophilic and fibrillary. In higher-grade tumors, cytologic atypia is common, associated with monster cells, normal and abnormal mitoses, foci of rhabdoid cytomorphology, and tumor necrosis. Several variants of LMS have been described, including the pleomorphic (10\% of cases), myxoid, inflammatory, epithelioid, and osteoclast-like cell/giantcell-rich LMS. EBV-associated LMS differs from classical LMS in several respects. They tend to be well differentiated and to exhibit reduced cytologic atypia, low mitotic activity, and numerous intratumoral lymphocytes.

Immunohistochemically, LMS show features of smooth muscle differentiation. Tumor cells are generally positive for muscle-specific actin (HHF-35), alpha smooth muscle actin, desmin, calponin, h-caldesmon, and smooth muscle

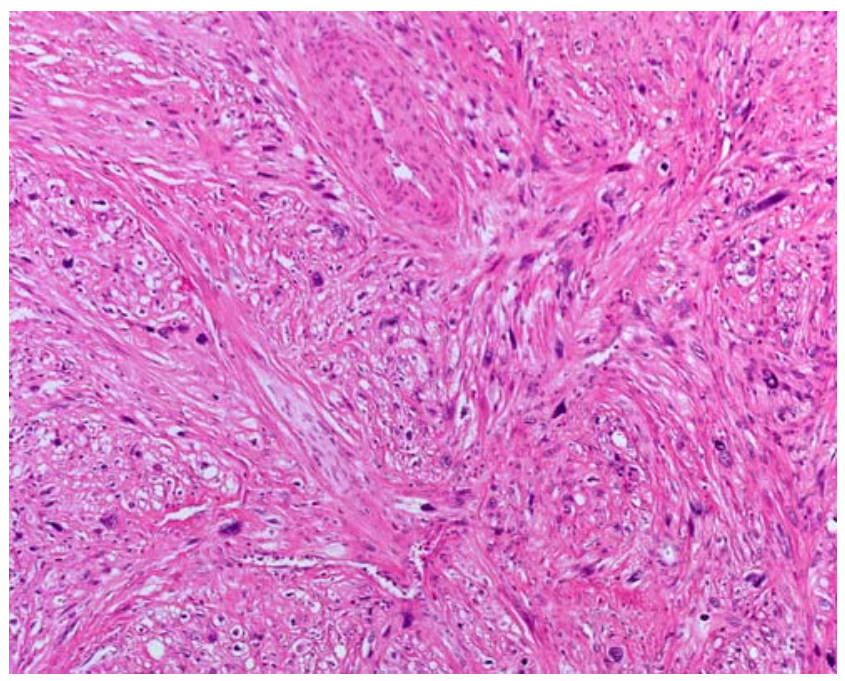

Fig. 1 Leiomyosarcoma. The tumor is moderately differentiated, composed of intersecting fascicles of spindle cells showing brightly eosinophilic cytoplasm and obvious nuclear atypia myosin heavy chain and are negative for myogenin. A significant proportion of LMS (especially welldifferentiated neoplasms) can be positive for EMA (10$30 \%$ ), keratin (10-30\%), and CD34 (30\%). Extrauterine LMS are generally negative for estrogen and/or progesterone receptors.

\section{Differential diagnosis}

The differential diagnosis of LMS is quite large. Among benign lesions, inflammatory myofibroblastic tumor and all pseudosarcomatous (myo)fibroblastic reactive lesions (e.g., postoperative spindle cell nodule) are most likely to be confused with LMS. Spindle cell malignant neoplasms resembling LMS include myofibroblastic sarcoma, spindle cell rhabdomyosarcoma, pleomorphic rhabdomyosarcoma, high-grade undifferentiated pleomorphic sarcoma, gastrointestinal stromal tumor, sarcomatoid carcinoma, and spindle cell/pleomorphic melanoma.

\section{Undifferentiated spindle/pleomorphic sarcoma (so-called storiform-pleomorphic MFH)}

Undifferentiated high-grade spindle/pleomorphic sarcoma (undifferentiated pleomorphic sarcoma (UPS)) is now the preferred term to designate high-grade soft tissue sarcomas which fail to show any specific line of differentiation using currently available ancillary techniques and in which epithelial, melanotic, and lymphoid differentiations have been excluded [1, 28, 29]. It is a diagnosis of elimination. These neoplasms account for about 5-7\% of sarcomas occurring in adults. Most were previously called "storiform-pleomorphic malignant fibrous histiocytomas".

\section{Cytogenetics and molecular genetics}

UPS show complex cytogenetic rearrangements involving $30-35 \%$ of the genome, but no specific structural or numerical abnormalities have been proven, so far, to be useful for identification purposes [30] (Fig. 2). UPS and other pleomorphic sarcomas share many of the aberrations observed in LMS [31-33], including losses of 1q32.1, 2p25.3, 2q36-q37, 8p23, 9p, 10q21-q23, 11q22, 13q14$\mathrm{q} 21,16 \mathrm{q} 11$, and 16q23, gains of 1p36-p31, 1q21-q24, 2p, 4p16, 5p, 5q34, 6q, 7p15-p22, 7q21-qter, 17q, 9q, 14q, $16 \mathrm{p} 13,17 \mathrm{q}, 19 \mathrm{p} 13,19 \mathrm{q} 13.11-\mathrm{q} 13.2,20 \mathrm{q}$, and $21 \mathrm{q}$, and high-level amplifications of loci 1p33-p34, 12q13-q15, 17cen-p11.2, and 17p13-pter (Figs. 3 and 4). Both tumor types share also very similar protein expression patterns $[16,34]$. Loss of chromosome $13 \mathrm{q}$ is the most frequent genomic imbalance in UPS [35], leading to inactivation of the RB pathway [36]. Alterations within the TP53/ARF/ 


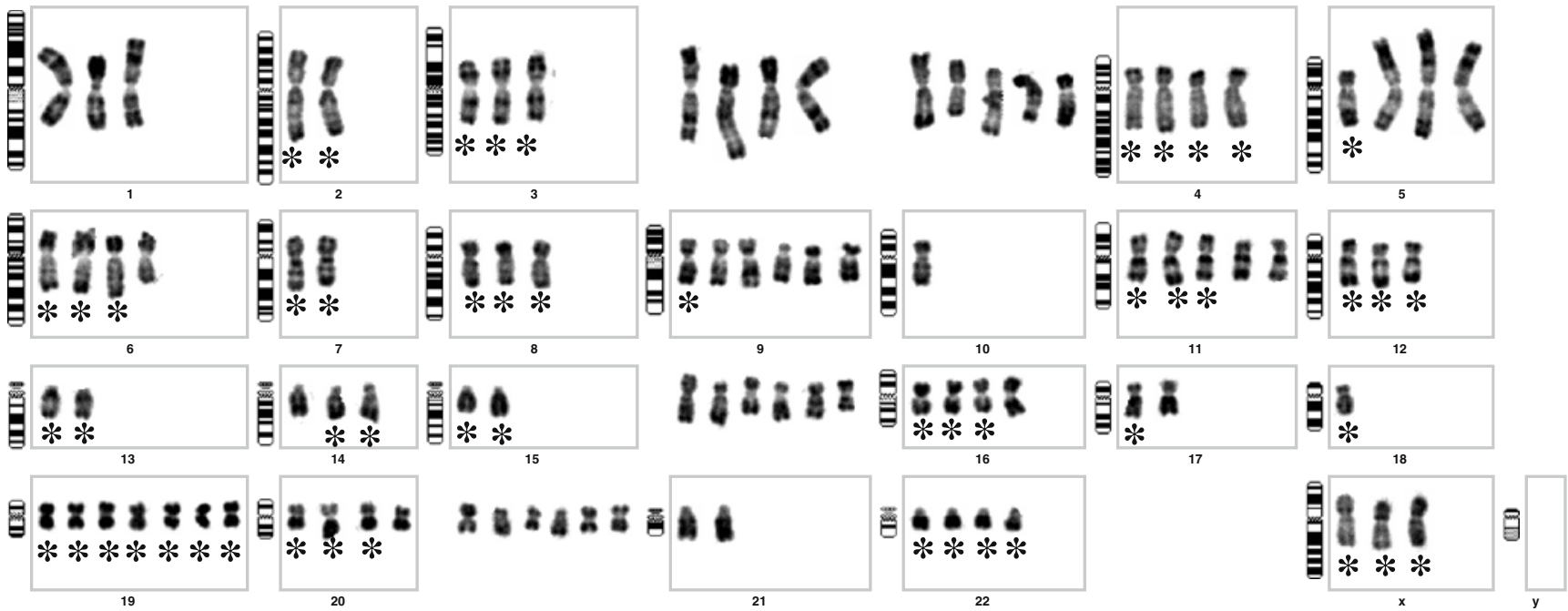

Fig. 2 Complex karyotype of an undifferentiated high-grade pleomorphic. sarcoma. Many chromosomes are abnormal and difficult to classify (asterisk indicates «apparently» normal (i.e., recognizable) chromosomes). Courtesy of Dr. F. Chibon, Bordeaux, France

MDM2 pathway are also frequent [32]. A recent gene expression analysis of 64 spindle and pleomorphic sarcomas showed that, when performing hierarchical clustering analyses, dedifferentiated liposarcoma, myxofibrosarcoma, leiomyosarcoma, malignant peripheral nerve sheath tumor, and adult-type fibrosarcoma formed their own clusters [37]. This study also showed that many of the so-called UPS/ MFH had heterogeneous profiles and could be reclassified
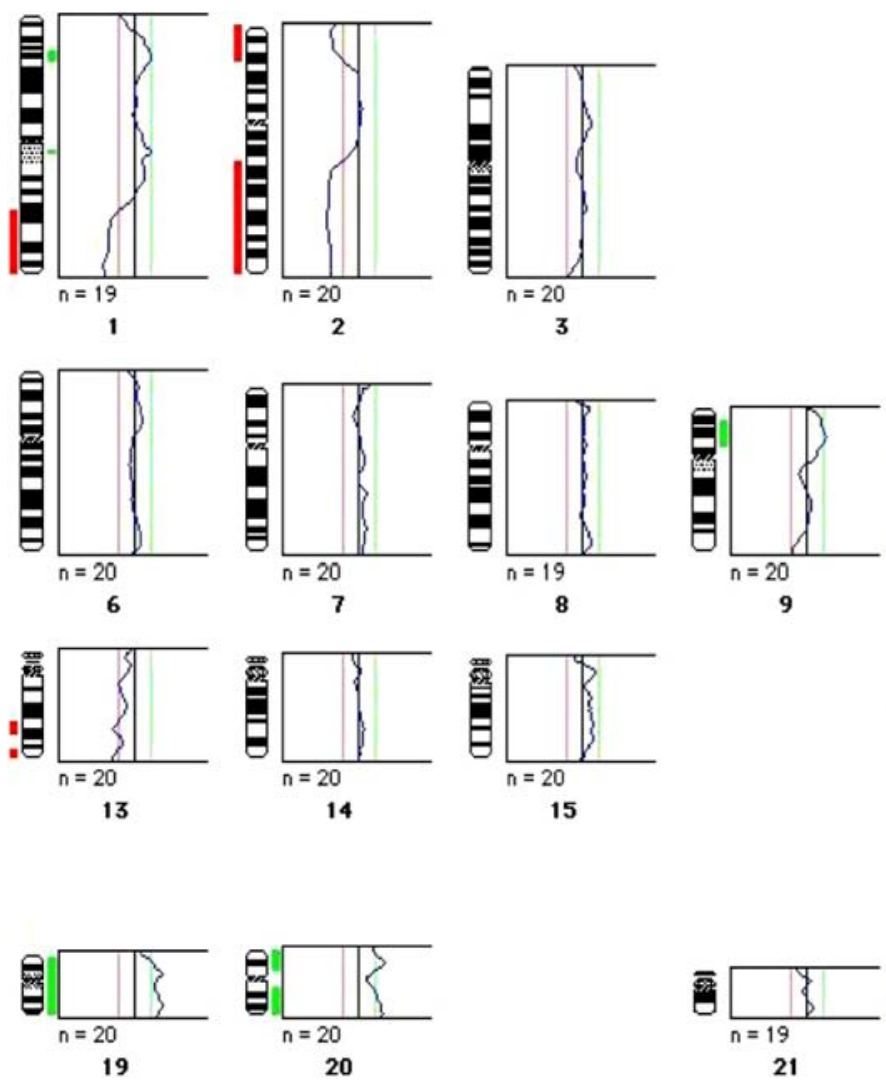
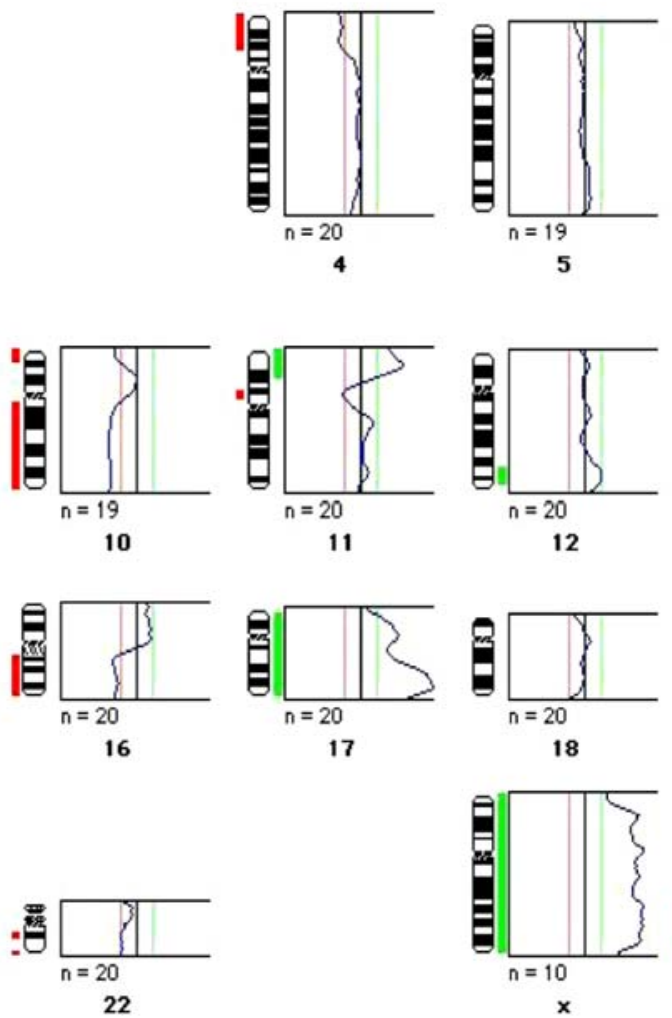

18
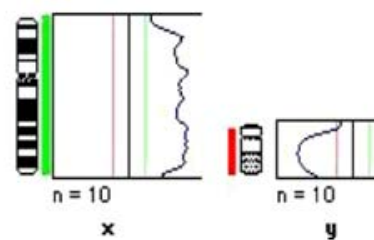

Fig. 3 Metaphase-based CGH of an undifferentiated high-grade pleomorphic sarcoma. Chromosomal imbalances are more easily depicted on CGH (chromosomal gains in green, losses in red) than on conventional karyotype. In this example, the tumor shows, among

other rearrangements, gains of chromosomes 19 and 20 and losses of 1qter, $10 \mathrm{q}, 13 \mathrm{q}$, and $16 \mathrm{q}$. This cytogenetic profile is not specific for undifferentiated pleomorphic sarcomas and can also be observed in leiomyosarcomas. Courtesy of Dr. F. Chibon, Bordeaux, France 

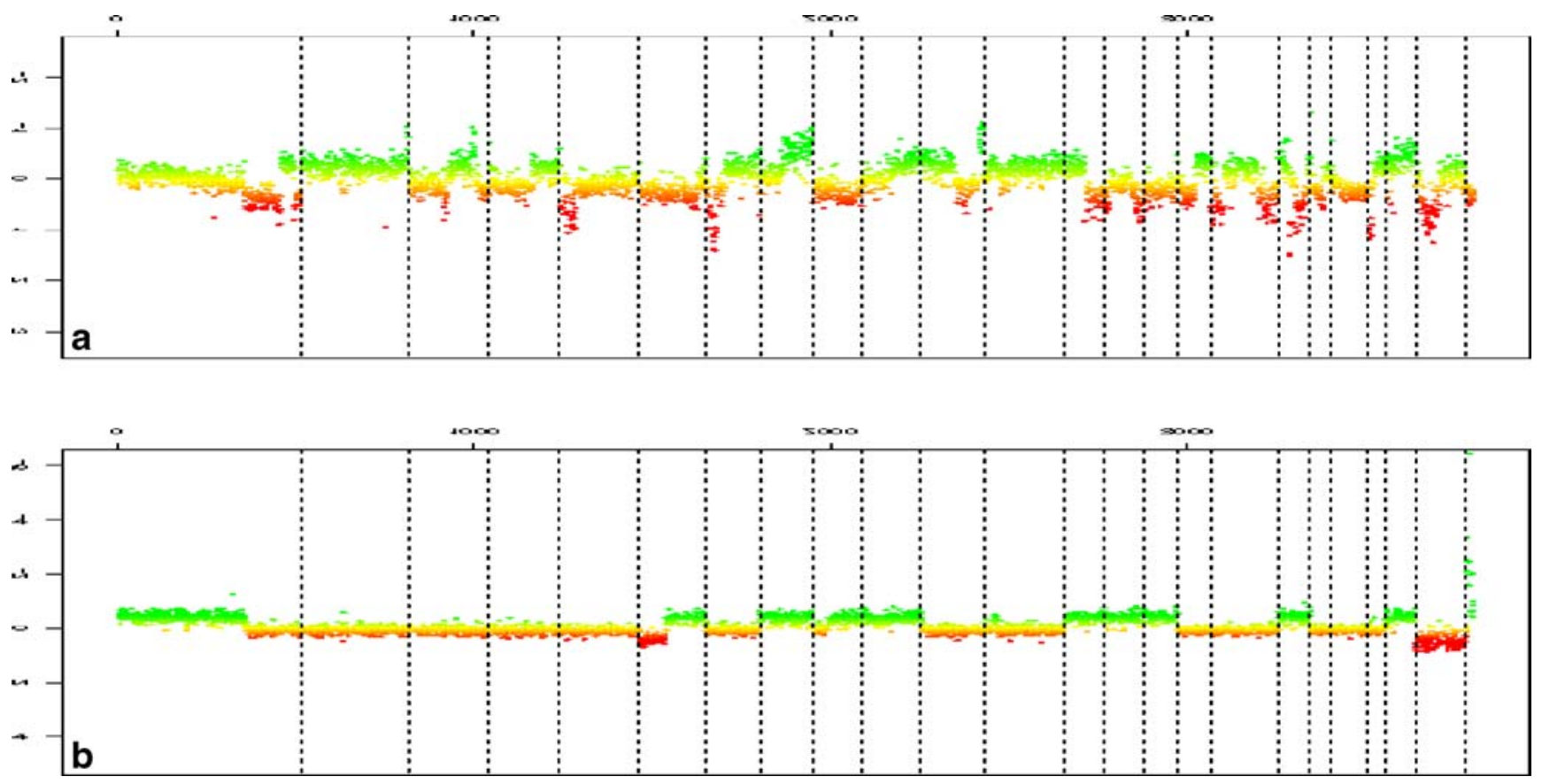

Fig. 4 Genomic profiles established by CGH BAC-array. This molecular approach allows us to identify rearranged chromosomal regions in undifferentiated pleomorphic sarcomas. Two types of genomic profiles are shown, one very complex (a) often associated with poor outcome and one less complex (b), associated (in the extremities) with better prognosis. Courtesy of Dr F. Chibon, Bordeaux, France into other histologic subcategories of pleomorphic sarcomas, especially in the myxofibrosarcoma category. Interestingly and, in some way reassuring, the authors performed a histopathologic review after their molecular analysis, and they found that morphologic features were essentially in accordance with their molecular results [37].

Matrix-CGH studies showed that the gene TRIO, coding for a guanidine nucleotide exchange factor, is upregulated in pleomorphic sarcomas, correlating with copy number gains and high-level amplifications of the short arm of chromosome 5 [10]. TRIO is implicated in the control of cytoskeleton organization, transcription regulation, cell cycle progression, apoptosis, vesicle trafficking, and cellto-cell adhesion, through activation of the Rho GTPasemediated signaling pathway [10]. As in LMS, TRIO seems to play a significant role in UPS progression [10].

Along with LMS, pleomorphic sarcomas also show RASSF1A hypermethylation [19] (albeit less than in LMS) and upregulation of several hypoxia-related genes (e.g., HIF1A and its targets) as well as of several genes involved in cell proliferation, adhesion, and motility, correlating with increased aggressiveness and/or increased metastatic potential [21, 38]. A recent analysis of 49 UPS and LMS, which confirmed the strong similarities between the two histotypes in terms of genomic aberrations and gene expression patterns, identified two, so far unrecognized, prognostic factors. One, loss of 4q31 (a region which encompasses the SMAD1 gene involved in the TGF-beta pathway), was a significant predictor of metastasis in multivariate analysis for the entire group of UPS and LMS, and the other, loss of $18 \mathrm{q} 22$, was a favorable prognostic factor for the same group [32].

Ezrin (villin 2), a protein that serves as an intermediate between the plasma membrane and the actin cytoskeleton, is considered as a marker of cancer progression and a potential target for cancer therapy. It plays a key role in cell morphology, adhesion, migration, and organization. Ezrin is overexpressed in many neoplasms, and this overexpression was found to correlate with increased metastatic potential and reduced survival [39]. Not surprisingly, this was also the case for high-grade sarcomas including UPS [40, 41].

The cell of origin of STS is a matter of controversy. Recently, it has convincingly shown that mesenchymal stem cells are good progenitor candidates for Ewing [42] and myxoid liposarcoma [43] development. Mesenchymal stem cells seem also to be good progenitors for $\mathrm{MFH}$ development via inactivation of the Wnt pathway [44]. Recently, Matushansky et al. [44] nicely demonstrated that DKK1, a Wnt inhibitor and mediator of human mesenchymal stem cell proliferation is overexpressed in UPS/MFH and that human mesenchymal stem cells can be transformed via inhibition of the Wnt signaling to form UPS/MFH-like tumors in nude mice. This undifferentiation (or "dedifferentiation") can be reversed if the Wnt signaling pathway is appropriately reestablished. 


\section{Clinical features}

UPS occurs most frequently in the limbs (especially lower limbs) and less often in the trunk of middle to advanced-aged adults, with a male predominance $[1,2]$. Less than $10 \%$ are observed in the subcutaneous fat, most present as deepseated masses. UPS are aggressive neoplasms; $30-50 \%$ of patients die within 5 years after diagnosis. Wide excision followed by irradiation is the treatment of choice. Adjuvant chemotherapy may be considered in selected cases (e.g., young patients). Myogenic differentiation is a negative prognostic factor in pleomorphic sarcomas [45, 46], with, however, the notable exception of dedifferentiated liposarcomas showing heterologous myogenic differentiation [47].

\section{Pathologic features}

UPS usually present as large $(5-15 \mathrm{~cm})$, well-circumscribed, deep-seated masses. Cut surface often show hemorrhagic, myxoid, and/or necrotic changes. The lesion is composed by an admixture of spindle and pleomorphic cells, set in a variably collagenized extracellular matrix (Fig. 5). Cellularity is variable, and cellular atypia, nuclear pleomorphism, mitoses, abnormal mitoses, areas of tumor necrosis, histiocyte-like cells, and foamy cells, as well as giant tumor cells with enlarged, polylobulated nuclei are commonly observed.

Immunohistochemically, it is common for undifferentiated high-grade pleomorphic sarcomas to show some limited foci of smooth muscle actin reactivity, but hcaldesmon, desmin, S100 protein, and epithelial markers are usually not expressed. If they are, the diagnosis of

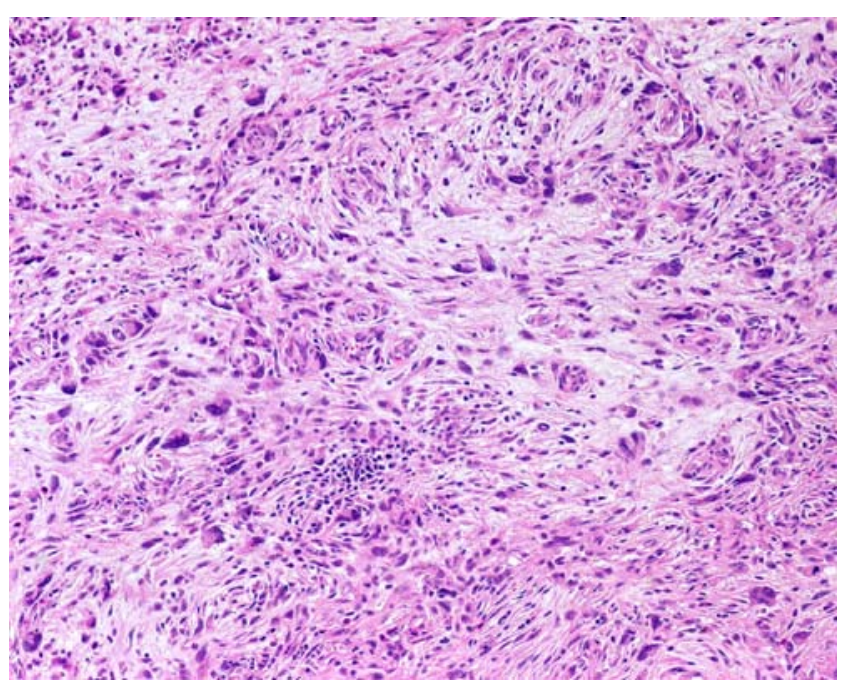

Fig. 5 Undifferentiated high-grade pleomorphic sarcoma. Proliferation of undifferentiated spindle and pleomorphic cells, set in variably collagenous background undifferentiated high-grade pleomorphic sarcoma should be questioned and a search for a specific line of differentiation pursued. UPS showing a significant amount of smooth muscle actin reactivity (and sometimes focal expression of desmin) but lacking the typical morphologic features of leiomyosarcoma are better classified as high-grade myofibrosarcomas (myofibroblastic sarcomas) or myosarcomas not otherwise specified [48, 49]. Histiocytic antigens (e.g., alpha-1-antitrypsin, alpha-1-antichymotrypsin, CD68, lysozyme) are of no utility. There are, by definition, no ultrastructural features of a specific lineage. Many tumor cells show features of fibroblasts, myofibroblast, or histiocytes.

\section{Differential diagnosis}

Undifferentiated high-grade pleomorphic sarcoma is a diagnosis of elimination [1, 28, 29]. Before giving such a diagnosis, all other potential mimics should have been ruled out, including metastatic carcinomas (lung, kidney), melanoma, lymphoma, pleomorphic rhabdomyosarcoma, pleomorphic leiomyosarcoma, pleomorphic liposarcoma, pleomorphic malignant peripheral nerve sheath tumor, liposarcoma with high-grade dedifferentiated areas, and high-grade myxofibrosarcoma. Extensive sampling, careful examination of slides (looking for lipoblasts or rhabdomyoblasts), and ancillary techniques (immunohistochemistry, electron microscopy, genetics) are critical in this context.

\section{Myxofibrosarcoma}

Myxofibrosarcoma, previously called myxoid malignant fibrous histiocytoma, is a relatively common sarcoma of older patients (median age 60 years) [1, 2, 50-53].

\section{Cytogenetics and molecular genetics}

Because myxofibrosarcoma has been, for a long time, included in the MFH category and because the separation between high-grade pleomorphic myxofibrosarcoma containing few $(<10 \%)$ myxoid areas and undifferentiated pleomorphic sarcoma (UPS) is difficult and somewhat subjective [1, 51, 52], data on the cytogenetics and molecular genetics of myxofibrosarcoma are limited. Myxofibrosarcomas are generally associated with very complex karyotypes, sharing many of the aberrations observed in LMS and UPS [30, 37, 54]. A recent clinicopathologic and karyotype analysis of 32 myxofibrosarcomas showed that local recurrence was associated with increase in tumor grade and in cytogenetic aberrations [55]. Chromosomal aberrations were not restricted to highergrade tumors but could also be observed in low-grade 
myxofibrosarcomas, albeit less frequently. Normal karyotypes were also observed, independently of grade.

A CGH analysis of a series of 22 myxofibrosarcomas showed recurrent simple gains of the chromosome regions $19 \mathrm{p}$ and $19 \mathrm{q}$, losses of chromosome 1q, 2q, 3p, 4q, 10q, $11 \mathrm{q}$, and 13q, and high-level amplifications of the central regions of chromosomes $1,5 p$, and 20q [54]. Loss of the 13q14-21 chromosomal region, encompassing the RB1 locus, was observed in all 22 cases examined. Of interest, gain of $5 p$ and loss of $4 q$ are not observed in low-grade myxofibrosarcomas as opposed to myxofibrosarcomas of higher grades, suggesting that these aberrations are late events in the oncogenesis of myxofibrosarcoma [54]. In their comparative study, the authors also found similar chromosomal aberrations in a series of nine pleomorphic liposarcomas, suggesting close relationship between myxofibrosarcoma and pleomorphic liposarcoma.

A recent gene expression analysis of 64 spindle and pleomorphic sarcomas showed that, when performing hierarchical clustering analyses, myxofibrosarcoma could be separated from other spindle cell/pleomorphic sarcomas, namely dedifferentiated liposarcoma, leiomyosarcoma, malignant peripheral nerve sheath tumor, and fibrosarcoma. This study also showed that many neoplasms which had initially been (mis)classified as UPS/MFH based on their morphology and immunoprofile could be reclassified as myxofibrosarcoma based on gene expression [37]. Two genes, GPR64 and TNXB, were particularly expressed by myxofibrosarcomas but not by UPS/MFH, thus allowing distinction between the two histotypes [37]. Separating a low-grade myxofibrosarcoma from a cellular myxoma can be difficult. A recent study showed that this can be done based on the differences in genetic alterations and in the composition of extracellular matrix [56].

\section{Clinical features}

Myxofibrosarcoma is mostly found in the deep dermis and subcutaneous fat of limbs (especially lower limbs) and limb girdles $[1,2,50-53]$. Thirty percent to $60 \%$ of the cases are deep-seated, developing in fascia and skeletal muscle. This is a slow-growing, often painless, tumor affecting men and women equally. Clinical behavior depends on tumor size, histologic grade (which includes tumor necrosis, mitotic rate, and amount of myxoid areas), and extent of resection $[51,52]$. Local recurrences occur in about $50 \%$ of cases, often because of inadequate initial excisions. It is not unusual for recurrences to show signs of upgrading with an increase in cellularity, pleomorphism and mitotic activity, and, conversely, a reduction of the myxoid component. Metastases to lungs and bone are mostly observed in highgrade, large deep-seated tumors (20-35\%); they are rare in low-grade lesions. The overall 5-year survival rate is 60
$70 \%$. A variant of myxofibrosarcoma showing distinctive epithelioid morphology and aggressive behavior has recently been individualized [57].

Pathologic features

Grossly, myxofibrosarcoma is typically ill-defined, heterogeneous, often multinodular, located in the subcutaneous fat, growing along fibrous septa and forming more or less gelatinous nodules. Large tumors are often deep-seated and partially necrotic and/or hemorrhagic. On microscopic examination, it is characterized by myxoid areas which are associated with cellular areas resembling undifferentiated pleomorphic sarcoma (Fig. 6). There is unfortunately no consensus on the extent of the myxoid areas required for the diagnosis of myxofibrosarcoma; whereas some authors require at least $50 \%$ [2] or $30 \%$ [52], $10 \%$ is enough for some others $[51,53]$. Low-, intermediate-, and high-grade tumors have been described, depending on the amount of cellular atypia and on the respective proportions of myxoid and nonmyxoid components (the latter showing morphologic features of storiform-pleomorphic malignant fibrous histiocytoma) [2]. Low-grade myxofibrosarcomas are predominantly myxoid $(\geq 50 \%$ myxoid) and hypocellular and contains distinctive curvilinear vessels (Fig. 7). Tumor cells, some of which have enlarged hyperchromatic nuclei, tend to aggregate around vessels. Vacuolated cells containing acid mucin and resembling lipoblasts are also found. Mitotic figures are rare. In high-grade lesions, the malignant fibrous histiocytoma-like component predominates: nuclear pleomorphism is evident, multinucleated giant cells and necrosis common, and mitotic figures, including abnormal mitoses, readily visible. Epithe-

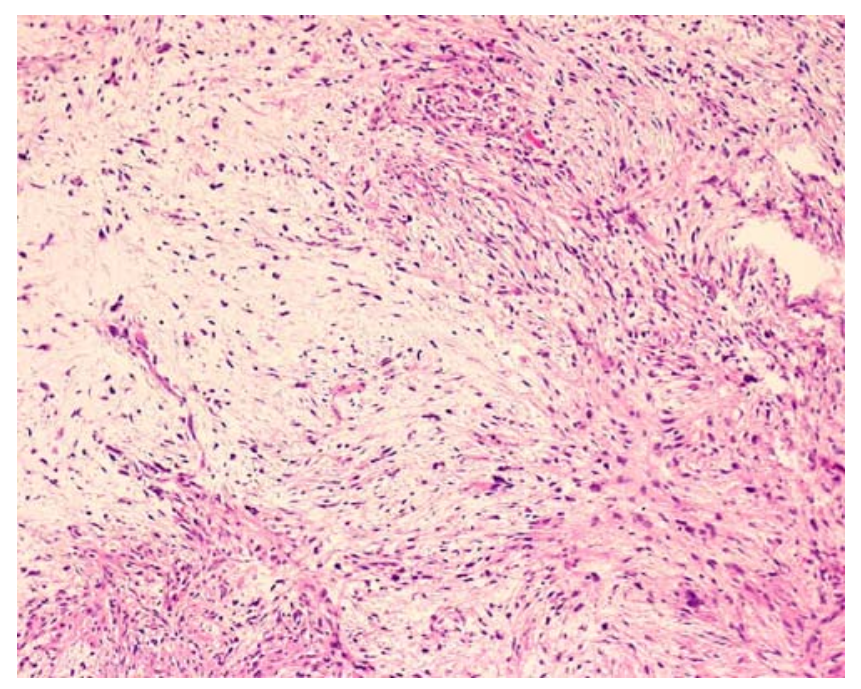

Fig. 6 Myxofibrosarcoma. This intermediate-grade myxofibrosarcoma is composed of an admixture of myxoid and cellular areas. Nuclear atypia are obvious 


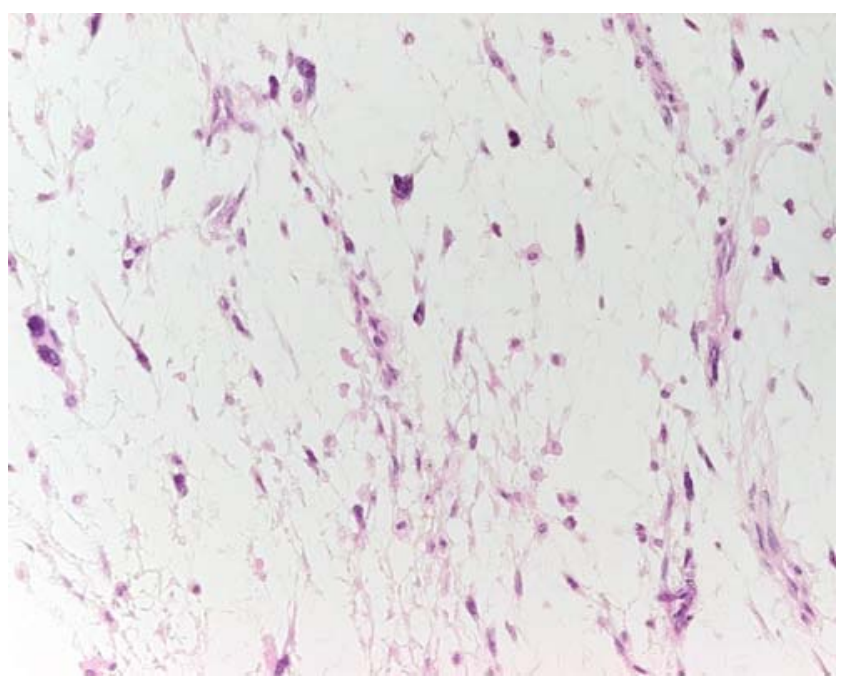

Fig. 7 Myxofibrosarcoma. This low-grade myxofibrosarcoma is paucicellular and contains an abundant myxoid extracellular matrix. Curvilinear vessels are readily visible. Despite low cellularity, nuclear atypia are always present

lioid myxofibrosarcomas are mostly high-grade tumors displaying prominent carcinoma-like or rhabdomyosarcomalike appearances [57]. Intermediate-grade myxofibrosarcomas are more cellular than low-grade lesions and often contain minute solid areas showing frank pleomorphism.

Immunohistochemically, tumor cells stain diffusely for vimentin and occasionally for smooth muscle actin. They are negative for S100 protein and CD34. They show ultrastructural features of fibroblasts and, less frequently, of myofibroblasts.

\section{Differential diagnosis}

High-grade myxofibrosarcoma may be confused with any pleomorphic sarcoma including myxoinflammatory fibroblastic sarcoma (see below). Occurrence in the elderly, subcutaneous location, and the presence of small myxoid areas in an otherwise pleomorphic tumor should point to the diagnosis. Epithelioid myxofibrosarcoma should be distinguished from a poorly differentiated carcinoma, a melanoma, a pleomorphic liposarcoma, and a pleomorphic rhabdomyosarcoma [57].

\section{Pleomorphic liposarcoma}

PLPS is the less common variant of liposarcoma, accounting for less than $5 \%$ of all liposarcomas. It tends to occur in elderly people (median age 55-65 years) [1, 2, 58-60].

Cytogenetics and molecular genetics

Data on the cytogenetics and molecular genetics of PLPS are relatively limited. PLSP show, characteristically, high chromosome counts and complex structural arrangements, similar to that observed in UPS [61]. No specific genetic alterations have been attributed to PLPS. CGH analyses showed gains of $1 \mathrm{p}, 1 \mathrm{q} 21-\mathrm{q} 32,2 \mathrm{q}, 3 \mathrm{p}, 3 \mathrm{q}, 5 \mathrm{p} 12-\mathrm{p} 15,5 \mathrm{q}$, $6 \mathrm{p} 21,7 \mathrm{p}, 7 \mathrm{q} 22,8 \mathrm{q}, 10 \mathrm{q}, 12 \mathrm{q} 12-\mathrm{q} 24,13 \mathrm{q}, 14 \mathrm{q}, 15 \mathrm{q}, 17 \mathrm{p}, 17 \mathrm{q}$, 18p, 18q12, 19p12, 19q13, 20q, 22q, and Xq21-q27, and losses of 1q, 2q, 3p, 4q, 10q, 11q, 12p13, 13q14, 13q21-qter, 14q23-24, 16q22, 17p13, 17q11.2, and 22q13 [54,62, 63]. Idbaih et al. [54] observed that chromosome imbalances encountered in PLPS were very similar to those observed in their series of myxofibrosarcoma, and that the two histotypes, taken together, can be distinguished from other pleomorphic sarcomas and more specifically from LMS. In the study of Schmidt et al. [62], patients with gains of 13q (i.e., 13q21, $13 \mathrm{q} 22,13 \mathrm{q} 31$, and 13q32) experienced shortened survivals as compared to those lacking these gains.

Deletion of 13q14.2-q14.3, targeting the RB1 pathway, is observed in about $60 \%$ of PLPS [63]. Other frequent events are loss of $17 \mathrm{p} 13$, spanning TP53, and loss of $17 q 11.2$ containing the neurofibromatosis type 1 (NF1) gene [63]. Mutations of TP53 are frequent in PLPS [61]. Amplification of $\delta$ catenin on $5 p$ and deregulation of genes involved in adipogenesis $(\mathrm{C} / \mathrm{EBP} \alpha$ and $\mathrm{C} / \mathrm{EBP} \gamma$ on $19 \mathrm{q}$, EP300 on 22q13) are also observed, and this may explain, in part, the propensity of PLPS to metastasize and to lose its adipocytic differentiation, respectively [63].

Despite their morphologic similarity, PLPS and highgrade dedifferentiated LPS show distinct chromosomal imbalances [64, 65]. Gains of 5p13-p15, 1p21, 1q21-q22, and $7 \mathrm{q} 22$ are more frequently observed in PLPS whereas high-level amplifications within chromosomal subregion 12q13-q21 were observed only in dedifferentiated LPS [64, 65]. Using a microarray-based gene expression profiling approach, Singer et al. [66] show that dedifferentiated and pleomorphic liposarcomas had different molecular signatures. Distinction between these two histotypes can be performed based on 15 differentially expressed genes, of which 12 (e.g., MDM2, CDK4) were located in the 12p13p15 region [66]. Similar results were obtained by Fritz et al. in 2002 [65]. MAD2, a gene involved in the RB1 signaling pathway which is overexpressed by PLPS (and dedifferentiated LPS), might constitute a therapeutic target [66].

Recently, Matushansky et al. [67] proposed a nice hypothesis according to which soft tissue sarcomas, including LPS, could be categorized by their developmental/differentiation status from stem cell to mature tissue. Gene expression analyses were performed during in vitro differentiation of human mesenchymal stem cells into adipose tissue and results compared to gene expression in each of the four liposarcoma subtypes. The authors observed that some genes that were overexpressed in a given liposarcoma subtype were also overexpressed during given stages of normal adipocytic differentiation. In other 
terms, each liposarcoma subtype profile would indicate the stage of differentiation arrest. After excluding markers of differentiation, the authors observed that cell cycle and purine metabolism pathways were upregulated in PLPS and MAPK kinase and insulin signaling pathways downregulated, suggesting that these pathways play significant role in PLPS tumorigenesis. Interestingly and unexpectedly, KRAS (a well-characterized oncogene) is overexpressed in PLPS as a marker of differentiation and not as a marker of tumorigenesis. They also observed that some liposarcoma subtypes do not parallel corresponding stages of adipocytic differentiation, suggesting additional molecular events [67].

\section{Clinical features}

PLPS occur predominantly in deep soft tissues $(75 \%$ of cases) of the lower extremities, especially the thigh $[1,2$, 58-60]. The limb girdles and the trunk (including the retroperitoneum) are each involved in $20 \%$ of cases. PLPS are high-grade tumors that recur locally in $35-50 \%$ and metastasize in $30-40 \%$ of cases. Five-year overall, metastasis-free and local recurrence-free survivals are 40 $63 \%, 30-58 \%$, and $25-75 \%$, respectively [1, 2, 58, 59]. Tumor grade and morphologic features (except for epithelioid morphology) seem not to affect patient outcome [5860]. Tumors $>5 \mathrm{~cm}$, deep-seated lesions, and truncal tumor location are adverse prognostic factors $[58,59]$. Although rare, dermal PLPS have a much better prognosis as they are easily amenable to complete excision [68].

\section{Pathological features}

PLPS are composed of a varying combination of lipogenic and nonlipogenic areas (Fig. 8). Lipogenic areas are composed of malignant adipocytes and/or pleomorphic, often multivacuolated, lipoblasts with scalloped hyperchromatic nuclei. Nonlipogenic areas are characterized by malignant fibrous histiocytoma-like, leiomyosarcoma-like, round-cellliposarcoma-like, and/or epithelioid/carcinoma-like features $[1,2,58-60]$. Areas showing hemangiopericytoma-like features are also occasionally observed [58, 59]. Tumor necrosis is common.

Immunohistochemically, lipogenic areas are often positive for S100 protein (35-50\% of cases), whereas nonlipogenic areas show varying and often focal staining for smooth muscle actin, desmin, and CD34. Focal reactivity for keratins and/or EMA is occasionally observed, especially in PLPS containing epithelioid areas [58-60].

\section{Differential diagnosis}

Diagnostic criteria for PLPS rely on the histologic presence of malignant adipocytes/lipoblasts in a high-grade pleomor-

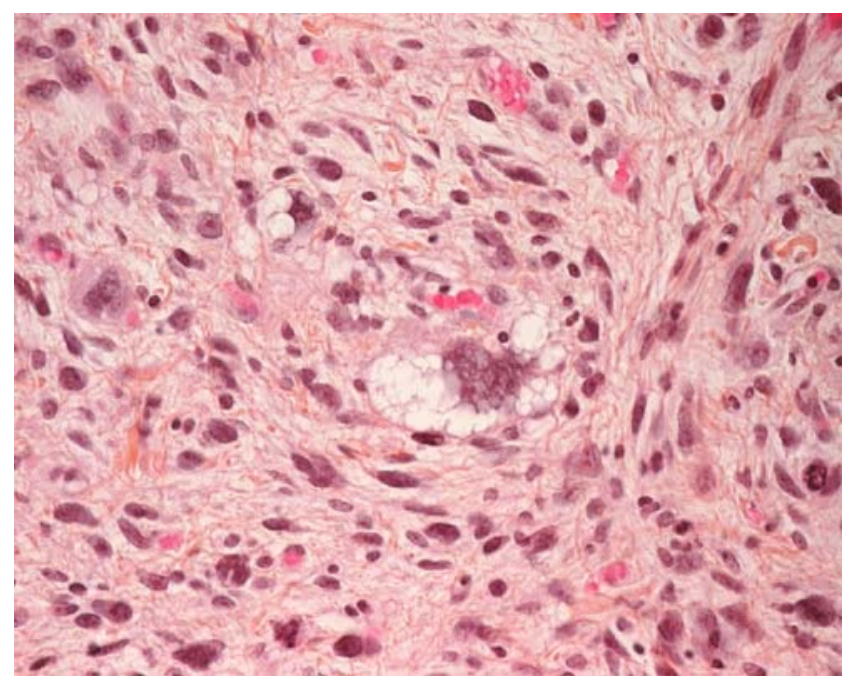

Fig. 8 Pleomorphic liposarcoma. The tumor shows the appearance of an undifferentiated pleomorphic sarcoma, except for the presence of highly pleomorphic and multivacuolated malignant adipocytes

phic sarcoma, regardless of the extent of lipogenic areas. As a consequence, any pleomorphic sarcoma enters the differential of PLPS, and adequate (extensive) sampling is crucial to pick up minute lipogenic foci. Melanoma and sarcomatoid carcinoma (renal cell carcinoma, adrenocortical carcinoma) should be ruled out. Cytoplasmic vacuolization resulting from fixation artifacts may be misleading, mimicking true lipoblasts. PLPS composed predominantly of small round cells may be confused with round cell liposarcoma (especially in the thigh). As opposed to PLPS, dedifferentiated LPS characteristically contains a juxtaposed well-differentiated liposarcomatous component and displays MDM2 and/or CDK4 nuclear reactivity.

\section{Pleomorphic rhabdomyosarcoma}

PRMS are rare neoplasms of adulthood. They account for about $5 \%$ of all adult pleomorphic soft tissue sarcomas $[1,2,69-72]$.

Cytogenetics and molecular genetics

Data on the cytogenetics and molecular genetics of PRMS are scarce. They show nonspecific complex karyotypes. Numerical and unbalanced structural abnormalities are common, overlapping with those of UPS or osteosarcoma, and are different from those encountered in embryonal and alveolar rhabdomyosarcoma. Chromosomal numerical aberrations include gains of chromosomes $1,5,8,14,18,20$, and 22 and losses of chromosomes 2, 5, 6, 10, 11, 13, 14, 15, 16, 17, 18,19 , and $\mathrm{Y}$, of which losses of chromosomes $2,13,14,15$, 16 , and 19 are the most frequent [73]. Using CGH, Gordon et 
al. [74] observed gains in the regions $1 \mathrm{p} 22-23,7 \mathrm{p}, 18 / 18 \mathrm{q}$, and 20/20p, losses of 10q23 (spanning the gene PTEN), 15q21-q22, 3p, 5q32-qter, and 13, and amplicons involving the regions 1p21-p31, 1q21-q25, 3p12, 3q26-qtel, 4q28-q31, $8 \mathrm{q} 21-\mathrm{q} 23 / 8 \mathrm{q}$, and 22q. With the exception of one case [74], PRMS do not contain the $\mathrm{t}(2 ; 13)$ or $\mathrm{t}(1 ; 13)$ alveolar rhabdomyosarcoma reciprocal translocations.

\section{Clinical features}

PRMS usually occur in the extremities, especially the thigh, of middle-aged adults (often men) [1, 2, 69-72], although a few cases have been observed in childhood [70, 72]. They are aggressive high-grade (grade 3) tumors, prone to recurrence and metastatic dissemination (lungs) [1, 2, 6972 ]. Roughly $70 \%$ of patients die of their disease within 2 years of diagnosis $[69,71,72]$.

\section{Pathologic features}

PRMS are essentially composed of large, pleomorphic, epithelioid to spindle-shaped cells with abundant, deeply eosinophilic cytoplasm and vesicular nuclei with prominent nucleoli. Marked nuclear atypia and tumor necrosis are common. Monstrous, often multinucleated, giant cells resembling rhabdomyoblasts are frequently encountered (Fig. 9), but cross-striations are rarely visible.

Immunohistochemically, tumor cells are positive for desmin and fast skeletal muscle myosin but also, at least focally, for smooth muscle actin. Because of poor specificity, myoglobin is not a good marker for the diagnosis of rhabdomyosarcoma. Myogenin reactivity is usually ob-

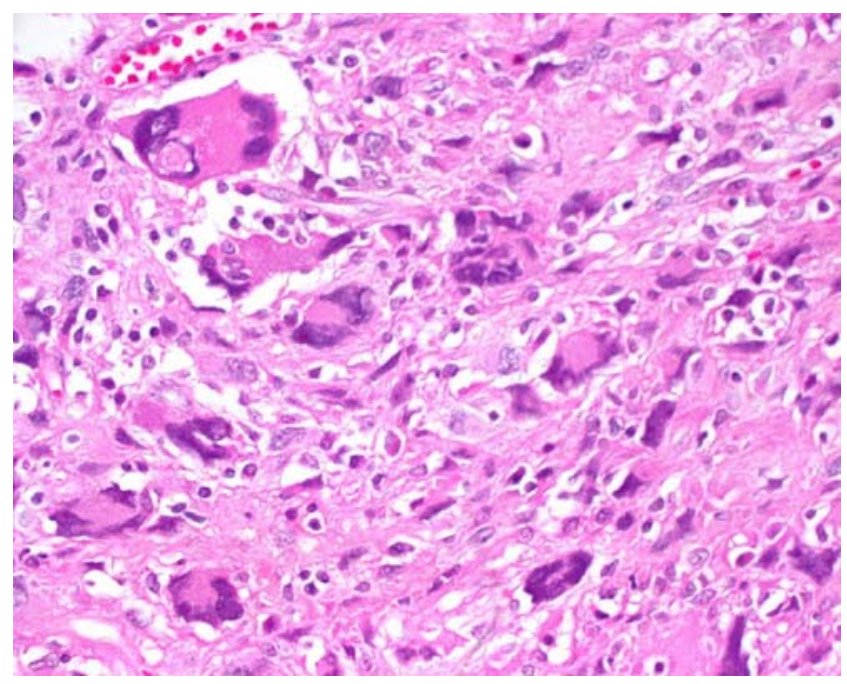

Fig. 9 Pleomorphic rhabdomyosarcoma. Intimate admixture of spindle cells and pleomorphic multinucleated giant cells corresponding to malignant rhabdomyoblasts served in a small proportion of tumor cells. Calponin (but not h-caldesmon) is positive. Rudimentary sarcomeres and ribosome-myosin complexes are observed in tumor cells on ultrastructural examination.

\section{Differential diagnosis}

The differential diagnosis for PRMS is similar to that of PLMS. Reactivity for desmin and myogenin are diagnostic clues. When faced with a tumor showing skeletal muscle differentiation, before considering the diagnosis of PRMS, other pleomorphic sarcoma subtypes that may contain foci of heterologous striated muscle differentiation should be ruled out, including dedifferentiated liposarcomas, malignant peripheral nerve sheath tumors (malignant Triton tumors), and metastases from metaplastic carcinomas (malignant mixed müllerian tumors) and Wilms' tumor.

\section{Malignant peripheral nerve sheath tumor}

MPNSTs are defined as malignant neoplasms arising from peripheral nerves or in extraneural soft tissue if it shows nerve sheath differentiation (i.e., Schwannian differentiation), excluding tumors originating from epineurial tissue or from peripheral nerve vasculature [75]. MPNSTs account for $5-8 \%$ of all soft tissue sarcomas; about $50 \%$ of them occur in the setting of NF1.

\section{Cytogenetics and molecular genetics}

Cytogenetically, MPNSTs display complex karyotypes and clonal chromosomal aberrations, regardless of clinical setting (i.e., sporadic versus NF1-associated MPNSTs). Chromosomal losses seem to be more frequent than gains [7, 76, 77]. Losses of 1p12-13, 1p21, 1p36, 3p21-pter, 9p13-21, 9p22-24, 10, 10p11-15, 11p, 11q21-25, 13q14, 15p, 16/16q24, 17/17p, 17q11-12, 17q21-25, 22, 22p, $22 q 13$, and 22q11-12 were the most frequent abnormalities observed [7, 76, 77]. Gains mainly involved the regions 7p21-q36, 7p22, 7q, 8, 8q11-23, 1q25-44, and 5q13-35. Breakpoints are often numerous, involving many chromosomes or chromosomal regions. Regions mostly involved are: $1 \mathrm{p}, 7 \mathrm{p} 22$ (spanning the ETV1 gene), 11q13-23, 20q13 (spanning the SRC gene), and 22q11-13 (spanning the NF2 gene) $[76,77]$. Ring chromosomes, trisomy 7 , and rearrangements of $11 \mathrm{p}$ and $12 \mathrm{q} 13-15$ were also reported [7]. Amplifications are rare [77]. In the series of Fletcher et al. [7], complex rearrangements were more frequent in MPNSTs associated with von Recklinghausen's disease and in tumors showing heterologous differentiation. There were no correlations between karyotypic abnormalities and clinical parameters [7]. Large and/or high-grade tumors 
frequently contain triploid or tetraploid clones, correlating with clinical aggressiveness [76]. Complex karyotypes are generally not observed in benign peripheral nerve sheath tumors (e.g., schwannoma and perineurioma), and this may help in classifying atypical peripheral nerve sheath neoplasms. Malignant Triton tumor and conventional MPNSTs lacking heterologous differentiation show similar complex karyotypes [77]. MPNSTs do not contain the $t(X ; 18)$ synovial sarcoma translocation.

In CGH analyses of MPNSTs, chromosomal gains tend to outnumber losses. Most frequent minimal recurrent regions of gain included 1q24-25, 8p23-p12, 9q34, and 17q23-q25 [78]. Gain of $17 q 23-25$ was strongly associated with poor prognosis, presence of metastases, and overexpression of some specific genes, namely topoisomerase II alpha, ETV4 (EIA), surviving/BIRC5 and HER2/neu (ERBB2) which might be involved in MPNST pathogenesis [78-81]. Gain of 7p15-p21 was also found associated with shortened survival [80]. Amplifications were also detected [82, 83].

Molecular genetics studies of MPNSTs showed that sporadic and NF1-associated MPNSTs display monoallelic or biallelic loss at the NF1 locus (localized at 17q11.2) in about $20-50 \%$ of cases [76, 83]. Germ line mutations of the NF1 gene, a tumor suppressor gene which encodes for the RasGTPase activating protein neurofibromin, have been demonstrated in families affected by von Recklinghausen disease. Loss of function of the NF1 gene (by mutation or loss of heterozygosity) is the key early step in the pathogenesis of peripheral nerve sheath tumors. It leads to neurofibromin loss and subsequent activation of the ras signaling cascade, increasing the risk of MPNST development [84]. Additional abnormalities are needed, however, to progress from benign to malignant peripheral nerve sheath tumor. TP53 (localized to 17p13) is frequently inactivated through mutations or deletions, correlating with the frequent loss of $17 \mathrm{p}$ [76]. The same observation applies to the regions 9 p21 and $13 q 14$ which are often rearranged or lost, resulting in an inactivation of the CDKN2A (encoding the $\mathrm{p} 16^{\mathrm{INK} 4 \mathrm{~A}}$ and $\mathrm{p} 14^{\mathrm{ARF}}$ cell cycle inhibitory proteins) and RB1 genes, respectively. The expression of $\mathrm{p} 15^{\mathrm{INK} 4 \mathrm{~B}}$ and $\mathrm{p} 27^{\mathrm{KIP} 1}$, two cyclin-dependent kinase inhibitors is also altered in MPNSTs [84]. The tumor suppressor NF2 gene is located at $22 \mathrm{q} 12.2$, and inactivation of this gene, mostly by mutations, has been demonstrated in neurofibromatosis type 2 . Since the region $22 q 11-13$ is frequently rearranged in MPNSTs, it has been hypothesized that the NF2 gene could play some role in the tumorigenesis and/or progression of MPNST [77]. Using highresolution DNA copy number profiling, Mantripragada et al. [83] recently reported amplifications of PDGFRA, ITGB4, MET TP73, HGF, and BIRC5/survivin and deletions of NF1, HMMR/RHAMM, MMMP13, CDKN2A, and TP53 in MPNSTs. Overexpression of
BIRC5/survivin correlates with an amplicon located at distal 17q [79]. Other recently identified genes such as TRIO, IRX2, and NKD2 [10] as well as TWIST1 and TNC [83] might also be implicated in MPNST pathogenesis.

\section{Clinical features}

Conventional MPNSTs occur mostly in middle- to advanced-aged adults, without sex predilection. Fifty percent to $70 \%$ develop in preexisting neurofibroma, especially in plexiform neurofibroma associated with NF1. Sporadic MPNSTs are less frequent, presenting as deep soft tissue masses, usually in association with large nerves (e.g., sciatic). Exceptionally, they may develop within a schwannoma. Ten percent of MPNSTs occur at a site of prior irradiation. NF1-associated MPNSTs occur generally 10 years younger than sporadic forms. Pain and/or neurological symptoms (e.g., paresthesias) are the most common presenting symptoms. Predominantly affected sites include the buttock and sacral plexus, thigh, paraspinal nerves, and brachial plexus [75].

Many clinicopathologic variants of MPNSTs have been described, including epithelioid MPNST (5\% of MPNSTs), MPNST with mesenchymal (osteosarcomatous, chondrosarcomatous, and/or angiosarcomatous) differentiation (10\% of cases), MPNST with perineurial differentiation, MPNST with rhabdomyosarcomatous differentiation (malignant Triton tumor), and glandular MPNST. Malignant Triton tumors and glandular MPNSTs are rare and associated with NF1 in $60 \%$ and $75 \%$ of cases, respectively. Epithelioid MPNSTs, which may occur either in the subcutis or in deep soft tissues, show no relationship to NF1.

Most MPNSTs are high-grade aggressive neoplasms, especially glandular and malignant Triton tumors. Outcome in MPNSTs depends on several factors, including tumor stage, tumor size, location, histologic subtype, status of surgical margins, previous irradiation, and high $(>25 \%)$ MIB-1 labeling index [75]. The prognostic value of histologic grade is limited in MPNSTs. About $60 \%$ of patients die of disease. Overall, 5- and 10-year survival rates are $34 \%$ and $23 \%$, respectively [75]. There are no significant prognostic differences between NF1-associated and sporadic MPNSTs.

Pathologic features

Most MPNSTs measure $5 \mathrm{~cm}$ or more in maximal diameter. High-grade tumors frequently contain areas of necrosis and/ or hemorrhage on cut section. Histologically, many MPNSTs present as monomorphic spindle cell tumors resembling adult-type fibrosarcoma or monophasic synovial sarcoma (Fig. 10). Alternating myxoid and cellular areas are frequently observed. Tumor cells tend to agglomerate 


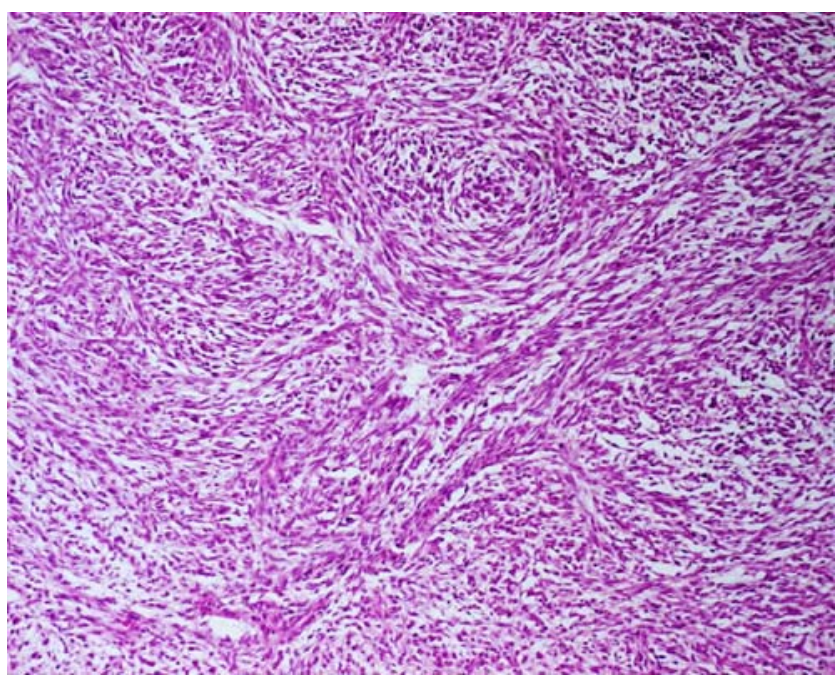

Fig. 10 Malignant peripheral nerve sheath tumor. The proliferation is composed of intersecting fascicles of monomorphic spindle cells. Some degree of whorling pattern is visible in this field

around large vessels, often infiltrating vessel walls. Tumor cell nuclei are typically buckled in well-differentiated lesions, resembling that observed in neurofibroma. Whorl structures, nuclear palisading, or reticular arrangement of tumor cells resembling perineurioma may be seen. Brisk mitotic activity and areas of geographic necrosis are common in large/high-grade neoplasms. Foci of osteosarcomatous and/ or chondrosarcomatous heterologous differentiation are observed in about $10 \%$ of tumors. About $5 \%$ of MPNSTs are characterized by prominent epithelioid cytomorphology. Rarely, MPNSTs show features of perineurial differentiation or contain rhabdomyoblasts (malignant Triton tumor; Fig. 11) or glandular epithelium. In glandular MPNSTs, benign or malignant-looking glandular structures are few in number, are well demarcated from the spindle cell component, and often contain mucinous (globet) cells and chromograninpositive endocrine cells. Squamous differentiation is very rare. Immunohistochemically, spindle cells in MPNSTs are focally reactive for $\mathrm{S} 100$ protein in $50 \%$ to $70 \%$ of cases. Staining is grade dependent; the higher the grade, the more patchy and less intense is the staining. They may also express CD57 and CD34, as well as EMA. EMA expression is usually taken as a sign of perineurial differentiation. MPNSTs are usually negative for cytokeratins. Most MPNSTs overexpress the P53 protein, in contrast to (nonatypical) neurofibromas. Focal reactivity for desmin and myogenin is seen in malignant Triton tumors. As opposed to conventional (e.g., spindle cell) MPNSTs, epithelioid MPNSTs are generally strongly and diffusely positive for S100 protein but negative for melanocytic and epithelial markers. Glandular structures in glandular MPNSTs express epithelial markers, CEA, and sometimes endocrine markers (chromogranin-A, CD56, synaptophysin).
Differential diagnosis

Conventional spindle cell MPNSTs should be first distinguished from monophasic synovial sarcoma and cellular schwannoma. Most spindle cell monophasic synovial sarcomas express EMA and/or cytokeratins, at least focally, and are negative for CD34. In addition, as opposed to MPNSTs, they bear the $\mathrm{t}(\mathrm{X} ; 18)(\mathrm{p} 11 ; \mathrm{q} 11)$ translocation which is specific of this tumor type. In addition, to be well circumscribed, cellular schwannomas are typically strongly and diffusely positive for S100 protein. MPNSTs should also be separated from other spindle cell tumors, namely leiomyosarcoma (negativity for S100 protein, reactivity for smooth muscle actin, h-caldesmon, and desmin), spindle cell rhabdomyosarcoma (positivity for desmin and myogenin), adult-type fibrosarcoma, spindle cell carcinoma, spindle cell melanoma (diffuse reactivity for $\mathrm{S} 100$ protein), and spindle cell mesothelioma. Glandular MPNST should be distinguished from biphasic synovial sarcoma and epithelioid MPNST from melanoma (positivity for HMB45, Melan-A, and/or Mitf), epithelioid sarcoma, epithelioid angiosarcoma, and epithelioid-looking myoepithelioma.

\section{Other soft tissue sarcomas with complex karyotypes}

\section{Adult-type fibrosarcoma}

Twenty years ago, fibrosarcoma was a common diagnosis. With the advent of immunohistochemistry and molecular ancillary techniques, numerous entities escape the illdefined group of fibrosarcoma (e.g., deep-seated fibromatosis, synovial sarcoma, malignant peripheral nerve sheath

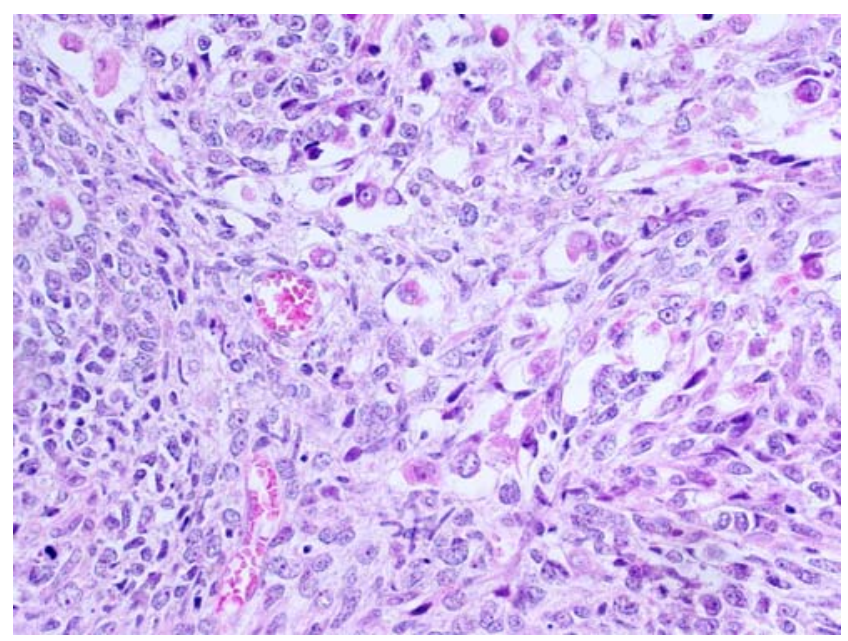

Fig. 11 Malignant Triton tumor. Small aggregates of relatively mature rhabdomyoblasts are observed, admixed with the spindle to round cell proliferation. The cytoplasm of rhabdomyoblasts is copious and markedly eosinophilic 
tumor, myofibroblastic sarcomas, dedifferentiated liposarcoma, dermatofibrosarcoma with fibrosarcomatous transformation, spindle cell rhabdomyosarcoma) whereas many others were recognized as specific fibrosarcomatous entities (e.g., infantile fibrosarcoma, low-grade fibromyxoid sarcoma, sclerosing epithelioid fibrosarcoma, myxoinflammatory fibroblastic sarcoma, myxofibrosarcoma, inflammatory fibrosarcoma). Nowadays, adult-type fibrosarcoma is a diagnosis of elimination. It accounts for no more than 3$5 \%$ of adult soft tissue sarcomas, and this sarcoma category overlaps morphologically with the undifferentiated spindle cell/pleomorphic sarcoma-UPS category $[1,2]$.

Because of the relative uncertainty about the diagnosis of adult-type fibrosarcoma, cytogenetic data are very scarce and questionable. Cytogenetically, adult fibrosarcoma shows multiple chromosomal rearrangements. Gains of 4q, 6q, 7, and 20 and loss of chromosomes 13 and 14 have been reported [7], as well as involvement of the 2q21qter region [1].

\section{Angiosarcoma}

Angiosarcomas are malignant neoplasms showing varying degrees of vascular differentiation. External beam radiation is an important risk factor for the development of angiosarcomas/lymphangiosarcomas of the skin, breast, and soft tissue. Other risk factors include exposure to vinyl chloride and thorium. Most angiosarcomas of soft tissue are composed by an admixture of spindle cells, epithelioid cells, and poorly formed vascular structures, although a predominantly epithelioid variant exists. Cytogenetic data are minimal for angiosarcoma. These tumors usually show complex karyotypes. The chromosomes number ranges from hypodiploid to hypertriploid. Most common chromosomal imbalances are gains of 5pter-p11, 8p12-qter, and 20pter-q12, losses of 4p, 7p15-pter, and 7p15-Y, and aberrations involving $22 \mathrm{q}[1,2]$. As opposed to Kaposi sarcoma, angiosarcomas of soft tissue are negative for HHV8. KRAS-2 and TP53 mutations were observed in thorium- and vinyl chloride-associated angiosarcomas as well as in sporadic angiosarcomas $[85,86]$. An alteration of the TP53/MDM2 pathway with elevated expression of TP53 and MDM2 proteins has been documented in $60 \%$ of angiosarcomas [87]. Loss of heterozygosity at TP53, WT1, and RB1 loci was recently reported in angiosarcomas, supporting a role for these tumor suppressor genes in angiosarcoma pathogenesis [88].

\section{Extraskeletal osteosarcoma}

Extraskeletal osteosarcoma is a malignant tumor showing osteoid formation. Most are high-grade neoplasms. It accounts for less than $5 \%$ of all osteosarcomas and occurs mostly in the deep soft tissue of middle-aged adults (median age 55-65 years) [89, 90]. Many of these tumors have been reported previously as giant cell malignant fibrous histiocytomas with bone production. The most commonly affected sites included lower extremities, especially the thigh $(50 \%$ of cases $)$, thoracic wall, and internal trunk [89, 90]. Half of neoplasms measured $5 \mathrm{~cm}$ or more in maximal diameter. As opposed to osseous osteosarcomas, extraskeletal osteosarcomas are relatively resistant to doxorubicin-based systemic therapy, and the prognosis is poor. Metastases develop in $50 \%$ to $60 \%$ of cases $[89,90]$. For patients with metastatic disease at diagnosis, median survival duration was 8 months in the series of Ahmad et al. [89]. For patients with localized disease at diagnosis, 5-year disease-specific and event-free survival rates were $46 \%$ and $47 \%$, respectively; quite similar to that observed in highgrade soft tissue sarcomas [89]. External beam irradiation is an acknowledged risk factor for the development of extraskeletal osteosarcoma [90, 91].

Morphologically, extraskeletal osteosarcoma resembles UPS except for the presence of osteoid (and sometimes chondroid) matrix.

Data on the cytogenetics of extraskeletal osteosarcoma are very scarce. Highly complex aberrations have been reported, including gains of 1q, 2, 8, and 17p11 and losses of 1q, 2, 5, 6, $12,13,14,15,16,18,19,20,21$, and Y [1, 30, 92]. These alterations parallel those observed in skeletal osteosarcoma, especially chromosomal gains and regional amplifications of chromosome arms 1q, 6p21-p12, 8q23-q24, and 17p13p11.2 [93, 94], partial or complete loss of $6 \mathrm{q}$, as well as various rearrangements of chromosomes 20 [95, 96]. TP53 (located at 17p13) and RB1 (located at 13q14) genes are consistently affected in skeletal and extraskeletal osteosarcomas (through allelic losses, rearrangements or point mutations), as well as many other genes involved in cell cycle regulation (p14 ${ }^{\mathrm{ARF}}, \mathrm{p} 16^{\mathrm{INKa}}, \mathrm{MDM} 2$, etc.) [97].

Myxoinflammatory fibroblastic sarcoma (inflammatory myxohyaline tumor)

Myxoinflammatory fibroblastic sarcoma is a low-grade neoplasm which is mostly (but not exclusively) observed in the distal extremities (50\% in fingers and hands) of middle-aged adults [1, 2, 98, 99]. The lesion, which is usually small (median 3-4 cm), develops in the subcutis, in deep soft tissues, or along tendon sheaths. Histologically, it bears a striking resemblance to high-grade myxofibrosarcoma, showing alternating myxoid, cellular, inflammatory, and fibrohyalinized areas (Fig. 12). Tumor cells are either spindle-shaped or epithelioid. Epithelioid cells tend to display a copious eosinophilic cytoplasm, enlarged vesicular nuclei, and macronucleoli, thus resembling ganglion cells or virocytes (Fig. 13). Myxoid areas often contain 


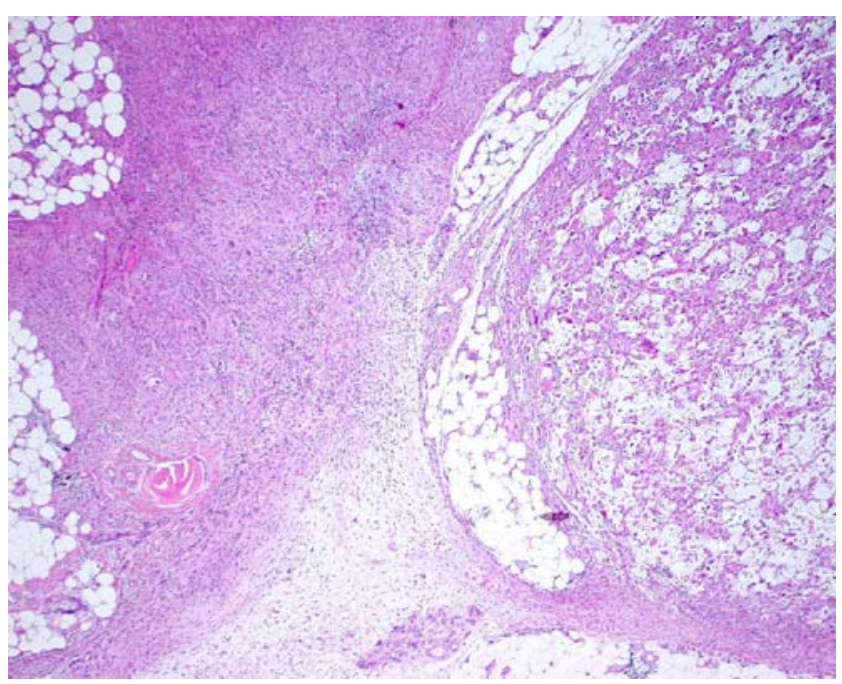

Fig. 12 Myxoinflammatory fibroblastic sarcoma (inflammatory myxohyaline tumor). An infiltrating neoplasm composed of spindle and pleomorphic cells, inflammatory elements, hypocellular to hyalinized areas, and myxoid areas containing vacuolated, lipoblast-like cells

univacuolated or multivacuolated lipoblast-like cells. Mitotic activity is usually low and tumor necrosis infrequent. Local recurrences are observed in about $20 \%$ of cases; metastases are rare $(<5 \%)$.

Most myxoinflammatory fibroblastic sarcomas show complex, near-diploid, or hypotriploid karyotypes [100103]. CGH analyses showed gains of chromosome 7 in two cases [101]. Distinctive abnormalities in myxoinflammatory fibroblastic sarcoma are the frequent presence of a balanced or unbalanced $\mathrm{t}(1 ; 10)(\mathrm{p} 22 ; \mathrm{q} 24)$ translocation [100-102], ring chromosomes [102, 103], and amplification of the chromosomal region 3p11-12 [103]. A $\mathrm{t}(2 ; 6)(\mathrm{q} 31 ; \mathrm{p} 21.3)$ translocation has also been described as a sole cytogenetic abnormality in this tumor type [104]. The breakpoints in the $t$ $(1 ; 10)$ translocation map to TGFBR3 in 1 p22 and in or near MGEA5 in 10q24, resulting in transcriptional upregulation of NPM3 and particularly FGF8, two consecutive genes located close to MGEA5 [103]. The ring chromosomes contain an amplified region in 3p11-12 which was associated with an increased expression of VGLL3 and CHMP2B. Beside typical examples of myxoinflammatory fibroblastic sarcoma, the amplicon in $3 p$ was also detected in some inflammatory variants of undifferentiated pleomorphic sarcoma (so-called inflammatory MFH) [103]. The $\mathrm{t}(1 ; 10)$ translocation was also observed in another tumor, the hemosiderotic fibrolipomatous tumor, which is likely to constitute a precursor for the myxoinflammatory fibroblastic sarcoma [103].

\section{Radiation-associated sarcomas}

Radiation-associated sarcomas are defined as sarcomas arising in a previously irradiated field after a latency period of at least 2 years $[91,105]$. Occurring in about $1 / 1,000$ patients who have undergone radiation therapy [106], it is an infrequent but well-known long-term complication of radiotherapy [91, 105-108]. They usually occur in middleaged adults and show a more aggressive clinical course associated with shortened patient survival as compared to sporadic sarcomas [91, 105-108]. Most frequent histologic types include high-grade undifferentiated pleomorphic sarcoma (previously called malignant fibrous histiocytoma), extraskeletal osteosarcoma, fibrosarcoma, malignant peripheral nerve sheath tumor, chondrosarcoma, leiomyosarcoma, and angiosarcoma [91, 105-108].

Radiation-associated soft tissue sarcomas are typically high-grade neoplasms with complex karyotypes. Numerical and structural (mostly unbalanced) rearrangements are common, irrespective of histological type [91]. Comparing postradiation and de novo sarcomas, Mertens et al. [91] observed that loss of material from chromosome $3 p$ (especially from the $3 \mathrm{p} 21-3$ pter region) was most frequent in postradiation sarcomas, in addition to the varying presence of cytogenetically unrelated clones. Other frequent imbalances were losses of Xq, 3p14, 1q42-qter, and 1q2324. CGH analyses disclosed gains of $5 \mathrm{p}, 7$ cen-q22, and 15 cen-q15 and loss of chromosome 13 [91]. The frequency of TP53 mutations is also very high as compared to that of sporadic sarcomas ( $88 \%$ versus $20 \%)$ [109].

\section{Conclusion}

Sarcomas displaying complex karyotypes are much more common than those harboring relatively simple cytogenetic

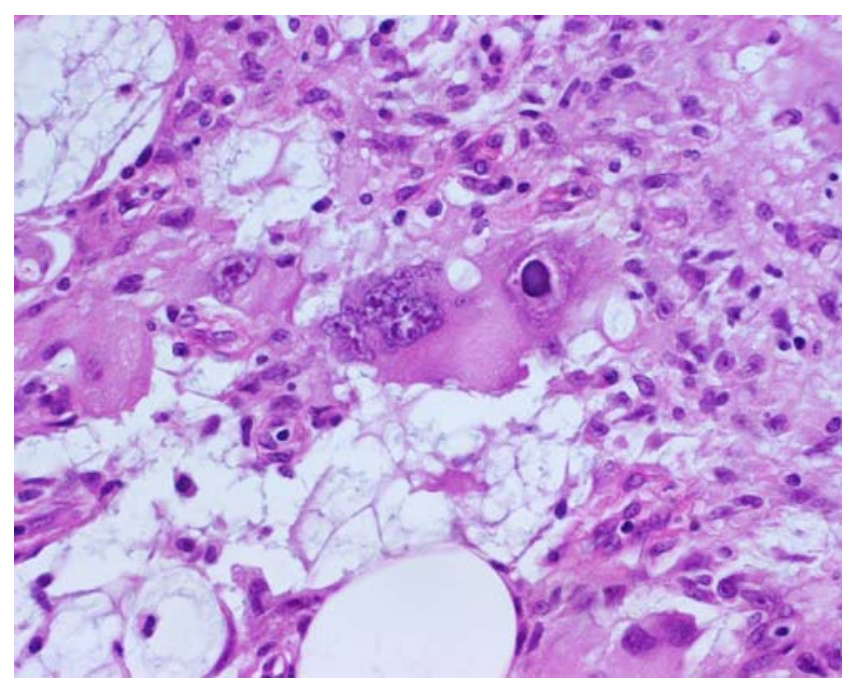

Fig. 13 Myxoinflammatory fibroblastic sarcoma (inflammatory myxohyaline tumor). Some pleomorphic cells contain viral-like intranuclear pseudoinclusions 
profiles with recurrent genetic aberrations (e.g., reciprocal translocations or mutations). Within the former group, leiomyosarcomas and undifferentiated pleomorphic sarcomas predominate. Thanks to new molecular technologies such as CGH-array-based techniques and gene expression analyses, significant progresses have been accomplished regarding the identification of genes, gene products, and signaling pathways particularly involved in the pathogenesis, progression, and metastatic dissemination of sarcomas. Novel diagnostic and/or prognostic molecular markers as well as promising therapeutic targets have been gradually recognized. A new era is coming in which candidates for adjuvant therapy will be selected not only on their clinicopathologic characteristics but also on their "molecular profile".

Conflict of interest statement We declare that we have no conflict of interest.

\section{References}

1. Fletcher CDM, Unni KK, Mertens F (eds) (2002) World Health Organization classification of tumours. Pathology and genetics of tumors of soft tissue and bone. IARC Press, Lyon

2. Weiss SW, Goldblum JR (2008) In: Weiss SW, Goldblum JR (eds) Enzinger and Weiss's soft tissue tumors, 5th edn. MosbyElsevier, Philadelphia

3. Dei Tos AP (2006) Classification of pleomorphic sarcomas: where are we now? Histopathology 48:51-62

4. Sandberg AA (2005) Updates on the cytogenetics and molecular genetics of bone and soft tissue tumors: leiomyosarcoma. Cancer Genet Cytogenet 161:1-19

5. Mandahl N, Fletcher CDM, Dal Cin P et al (2000) Comparative cytogenetic study of spindle cell and pleomorphic leiomyosarcomas of soft tissues: a report from the CHAMP Study Group. Cancer Genet Cytogenet 116:66-73

6. Yang J, Du X, Chen K et al (2009) Genetic aberrations in soft tissue leiomyosarcoma. Cancer Lett 275:1-8

7. Fletcher CDM, Dal Cin P, De Wever I et al (1999) Correlation between clinicopathologic features and karyotype in spindle cell sarcomas. A report of 130 cases from the CHAMP Study Group. Am J Pathol 154:1841-1847

8. Wang R, Titley JC, Lu YJ et al (2003) Loss of 13q14-q21 and gain of 5p14-pter in the progression of leiomyosarcoma. Mod Pathol 16:778-785

9. Hu J, Rao UNM, Jasani S et al (2005) Loss of DNA copy number of $10 \mathrm{q}$ is associated with aggressive behavior of leiomyosarcomas: a comparative genomic hybridization study. Cancer Genet Cytogenet 161:20-27

10. Adamowicz M, Radlwimmer B, Rieker RJ et al (2006) Frequent amplifications and abundant expression of TRIO, NKD2, and IRX2 in soft tissue sarcomas. Genes Chromosomes Cancer 45:829-838

11. Hernando E, Charytonowicz E, Dudas ME et al (2007) The AKT-mTOR pathway plays a critical role in the development of leiomyosarcomas. Nat Med 13:748-753

12. Bjornsti MA, Houghton PJ (2004) The TOR pathway: a target for cancer therapy. Nat Med 10:594-601
13. Mita MM, Tolcher AW (2007) The role of mTOR inhibitors for treatment of sarcomas. Current Oncology Reports 9:316-322

14. Lee YF, John M, Falconer A et al (2004) A gene expression signature associated with metastatic outcome in human leiomyosarcomas. Cancer Res 64:7201-7204

15. Ren B, Yu YP, Jing L et al (2003) Gene expression analysis of human soft tissue leiomyosarcomas. Hum Pathol 34:549-558

16. Suehara Y, Kondo T, Fujii K et al (2006) Proteomic signatures corresponding to histological classification and grading of softtissue sarcomas. Proteomics 6:4402-4409

17. Kawaguchi K, Oda Y, Saito T et al (2003) Mechanisms of inactivation of the p16INK4a gene in leiomyosarcoma of soft tissue: decrease. J Pathol 201:487-495

18. Dei Tos AP, Maestro R, Doglioni C et al (1996) Tumor suppressor genes and related molecules in leiomyosarcoma. Am J Pathol 148:1037-1045

19. Seidel C, Bartel F, Rastetter M et al (2005) Alterations of cancerrelated genes in soft tissue sarcomas: hypermethylation of RASSF1A is frequently detected in leiomyosarcoma and associated with poor prognosis in sarcoma. Int $\mathrm{J}$ Cancer 114:442-447

20. Kawaguchi K, Oda Y, Saito T et al (2006) DNA hypermethylation status of multiple genes in soft tissue sarcomas. Mod Pathol 19:106-114

21. Francis P, Namlos HM, Müller C et al (2007) Diagnostic and prognostic gene expression signatures in 177 soft tissue sarcomas: hypoxia-induced transcription profile signifies metastatic potential. BMC Genomics 8:73

22. Pérot G, Derré J, Coindre JM et al (2009) Strong smooth muscle differentiation is dependent on myocardin gene amplification in most human retroperitoneal leiomyosarcomas. Cancer Res 69:2269-2278

23. Deyrup AT, Lee VK, Hill CE et al (2006) Epstein-Barr virusassociated smooth muscle tumors are distinctive mesenchymal tumors reflecting multiple infection events: a clinicopathologic and molecular analysis of 29 tumors from 19 patients. Am J Surg Pathol 30:75-82

24. Kubben FJGM, Kroon FP, Hogendoorn PCW et al (1997) Absence of Epstein-Barr virus in a gastrointestinal stromal cell tumour (GIST) in an adult human immunodeficiency virusseropositive patient with past Epstein-Barr virus (EBV) infection. Eur J Gastroent Hepatol 9:721-724

25. Moinfar F, Azodi M, Tavassoli FA (2007) Uterine sarcomas. Pathology 39:55-71

26. Quade BJ, Wang TY, Sornberger K et al (2004) Molecular pathogenesis of uterine smooth muscle tumors from transcriptional profiling. Genes Chromosomes Cancer 40:97-108

27. Miettinen M, Fetsch JF (2006) Evaluation of biological potential of smooth muscle tumours. Histopathology 48:97-105

28. Fletcher CD (1992) Pleomorphic malignant fibrous histiocytoma: fact or fiction? A critical reappraisal based on 159 tumors diagnosed as pleomorphic sarcoma. Am J Surg Pathol 16:213228

29. Fletcher CDM (2006) The evolving classification of soft tissue tumours: an update based on the new WHO classification. Histopathology 48:3-12

30. Mertens F, Fletcher CDM, Dal Cin P et al (1998) Cytogenetic analysis of 46 pleomorphic soft tissue sarcomas and correlation with morphologic and clinical features: a report of the CHAMP Study Group. Genes Chromosomes Cancer 22:16-25

31. Derré J, Lagacé R, Nicolas A et al (2001) Leiomyosarcomas and most malignant fibrous histiocytomas share very similar comparative genomic hybridization imbalances: an analysis of a series of 27 leiomyosarcomas. Lab Invest 81:211-215

32. Carneiro A, Francis P, Bendahl PO et al (2009) Indistinguishable genomic profiles and shared prognostic markers in undifferenti- 
ated pleomorphic sarcoma and leiomyosarcoma: different sides of a single coin? Lab Invest 89:668-675

33. Larramendy ML, Gentile M, Soloneski S et al (2008) Does comparative genomic hybridization reveal differences in DNA copy number sequence patterns between leiomyosarcoma and malignant fibrous histiocytoma? Cancer Genet Cytogenet 187:1-11

34. Kawai A, Kondo T, Suehara Y et al (2008) Global proteinexpression analysis of bone and soft tissue sarcomas. Clin Orthop Relat Res 466:2099-2106

35. Mairal A, Terrier P, Chibon F et al (1999) Loss of chromosome 13 is the most frequent genomic imbalance in malignant fibrous histiocytomas: a comparative genomic hybridization analysis of a series of 30 cases. Cancer Genet Cytogenet 111:134-138

36. Chibon F, Mairal A, Fréneaux P et al (2000) The RB1 gene is the target of chromosome 13 deletions in malignant fibrous histiocytoma. Cancer Res 60:6339-6345

37. Nakayama R, Nemoto T, Takahashi H et al (2007) Gene expression analysis of soft tissue sarcomas: characterization and reclassification of malignant fibrous histiocytoma. Mod Pathol 20:749-759

38. Shintani K, Matsumine A, Kusuzaki K et al (2006) Expression of hypoxia-inducible factor (HIF)-I alpha as a biomarker of outcome in soft tissue sarcomas. Virchows Arch 449:673-681

39. Hunter KW (2004) Ezrin, a key component in tumor metastasis. Trends Mol Med 10:201-204

40. Kim MS, Cho WH, Song WS et al (2007) Prognostic significance of ezrin expression in pleomorphic malignant fibrous histiocytoma. Anticancer Res 27:1171-1178

41. Weng WH, Ahlén J, Aström K et al (2005) Prognostic impact of immunohistochemical expression of ezrin in highly malignant soft tissue sarcomas. Clinical Cancer Res 11:6198-6204

42. Riggi N, Cironi L, Provero P et al (2005) Development of Ewing's sarcoma from bone-marrow-derived mesenchymal progenitor cells. Cancer Res 65:11459-11468

43. Riggi N, Cironi L, Provero P et al (2006) Expression of the FUSCHOP fusion protein in primary mesenchymal progenitor cells gives rise to a model of myxoid liposarcoma. Cancer Res 66:7016-7023

44. Matuschansky I, Hernando E, Socci ND et al (2007) Derivation of sarcomas from mesenchymal stem cells via inactivation of the Wnt pathway. J Clin Invest 117:3248-3257

45. Fletcher CD, Gustafson P, Rydholm A et al (2001) Clinicopathologic re-evaluation of 100 malignant fibrous histiocytomas: prognostic relevance of subclassification. J Clin Oncol 19:3045-3050

46. Deyrup AT, Haydon RC, Huo D et al (2003) Myoid differentiation and prognosis in adult pleomorphic sarcomas of the extremity: an analysis of 92 cases. Cancer 98:805-813

47. Bui Nguyen Binh M, Guillou L, Hostein I et al (2007) Dedifferentiated liposarcomas with divergent myosarcomatous differentiation developed in the internal trunk. A study of 27 cases and comparison to conventional dedifferentiated liposarcomas and leiomyosarcomas. Am J Surg Pathol 31:1557-1566

48. Montgomery E, Fisher C (2001) Myofibroblastic differentiation in malignant fibrous histiocytoma (pleomorphic myofibrosarcoma): a clinicopathological study. Histopathology 38:499-509

49. Fisher C (2004) Myofibrosarcoma. Virchows Arch 445:215-223

50. Merck C, Angervall L, Kindblom LG et al (1983) Myxofibrosarcoma. A malignant soft tissue tumor of fibroblastic-histiocytic origin. A clinicopathologic and prognostic study of 110 cases using multivariate analysis. Acta Pathol Microbiol Immunol Scand Suppl 282:1-40

51. Mentzel T, Calonje E, Wadden C et al (1996) Myxofibrosarcoma: clinicopathologic analysis of 75 cases with emphasis on the low grade variant. Am J Surg Pathol 20:391-405

52. Huang HY, Lal P, Qin J et al (2004) Low-grade myxofibrosarcoma: a clinicopathologic analysis of 49 cases treated at a single institution with simultaneous assessment of the efficacy of 3-tier and 4-tier grading systems. Hum Pathol 35:612-621

53. Lin CN, Chou SC, Li CF et al (2006) Prognostic factors of myxofibrosarcomas: implications of margin status, tumor necrosis, and mitotic rate on survival. J Surg Oncol 93:294-3043

54. Idbaih A, Coindre JM, Derré J et al (2005) Myxoid malignant fibrous histiocytoma and pleomorphic liposarcoma share very similar genomic imbalances. Lab Invest 85:176-181

55. Willems SM, Debiec-Rychter M, Szuhai K et al (2006) Local recurrence of myxofibrosarcoma is associated with increased in tumour grade and cytogenetic aberrations, suggesting a multistep tumour progression model. Mod Pathol 19:407-416

56. Willems SM, Mohseny AB, Balog C et al (2009) Cellular/ intramuscular myxoma and grade I myxofibrosarcoma are characterized by distinct genetic alterations and specific composition of their extracellular matrix. J Cell Mol Med 13:1291-1301

57. Nascimento AF, Bertoni F, Fletcher CDM (2007) Epithelioid variant of myxofibrosarcoma: expanding the clinicomorphologic spectrum of myxofibrosarcoma in a series of 17 cases. Am J Surg Pathol 31:99-105

58. Gebhard S, Coindre JM, Michels JJ et al (2002) Pleomorphic liposarcoma: clinicopathologic, immunohistochemical, and followup analysis of 63 cases. A study from the French Federation of Cancer Centers Sarcoma Group. Am J Surg Pathol 26:601-616

59. Hornick JL, Bosenberg MW, Mentzel T et al (2004) Pleomorphic liposarcoma. Clinicopathologic analysis of 57 cases. Am J Surg Pathol 28:1257-1267

60. Miettinen M, Enzinger FM (1999) Epithelioid variant of pleomorphic liposarcoma: a study of 12 cases of a distinctive variant of high-grade liposarcoma. Mod Pathol 12:722-728

61. Sandberg AA (2004) Updates on the cytogenetics and molecular genetics of bone and soft tissue tumors. Liposarcoma. Cancer Genet Cytogenet 155:1-24

62. Schmidt H, Bartel F, Kappler M et al (2005) Gains of 13q are correlated with a poor prognosis in liposarcoma. Mod Pathol 18:638-644

63. Taylor BS, Barretina J, Socci ND et al (2008) Functional copynumber alterations in cancer. PLoS ONE 3:e3179

64. Rieker RJ, Joos S, Bartsch C et al (2002) Distinct chromosomal imbalances in pleomorphic and in high-grade dedifferentiated liposarcomas. Int J Cancer 99:68-73

65. Fritz B, Schubert F, Wrobel G et al (2002) Microarray-based copy number and expression profiling in dedifferentiated and pleomorphic liposarcoma. Cancer Res 62:2993-2998

66. Singer S, Socci ND, Ambrosini G et al (2007) Gene expression profiling of liposarcoma identifies distinct biological types/ subtypes and potential therapeutic targets in well-differentiated and dedifferentiated liposarcoma. Cancer Res 67:6626-6636

67. Matuschansky I, Hernando E, Socci ND et al (2008) A development model of sarcomagenesis defines a differentiation-based classification for liposarcomas. Am J Pathol 172:1069-1080

68. Dei Tos AP, Mentzel T, Fletcher CDM (1998) Primary liposarcoma of the skin: a rare neoplasm with unusual high grade features. Am J Dermatopathol 20:332-338

69. Furlong MA, Mentzel T, Fanburg-Smith JC (2001) Pleomorphic rhabdomyosarcoma in adults: a clinicopathologic study of 38 cases with emphasis on morphologic variants and recent skeletal muscle-specific markers. Mod Pathol 14:595-603

70. Furlong MA, Fanburg-Smith JC (2001) Pleomorphic rhabdomyosarcoma in children: four cases in the pediatric age group. Ann Diagn Pathol 5:199-206

71. Little DJ, Ballo MT, Zagars GK et al (2002) Adult rhabdomyosarcoma. Outcome following multimodality treatment. Cancer 95:377-388

72. Parham DM, Ellison DA (2006) Rhabdomyosarcomas in adults and children. An update. Arch Pathol Lab Med 130:1454-1465 
73. Li G, Ogose A, Kawashima $\mathrm{H}$ et al (2009) Cytogenetic and realtime quantitative reverse-transcriptase polymerase chain reaction analyses in pleomorphic rhabdomyosarcoma. Cancer Genet Cytogenet 192:1-9

74. Gordon A, McManus A, Anderson J et al (2003) Chromosomal imbalances in pleomorphic rhabdomyosarcomas and identification of the alveolar rhabdomyosarcoma-associated PAX3FOXO1A fusion gene in one case. Cancer Genet Cytogenet 140:73-77

75. Scheithauer BW, Louis DN, Hunter S et al (2007) Malignant peripheral nerve sheath tumour (MPNST). In: Louis DN, Ohgaki H, Wiestler OD, Cavenee WK (eds) World Health Organization classification of tumours of the central nervous system. IARC, Lyon, pp 160-162

76. Mertens F, Dal Cin P, De Wever I et al (2000) Cytogenetic characterization of peripheral nerve sheath tumours: a report of the CHAMP Study Group. J Pathol 190:31-38

77. Bridge RS, Bridge JA, Neff JR et al (2004) Recurrent chromosomal imbalances and structurally abnormal breakpoints within complex karyotypes of malignant peripheral nerve sheath tumour and malignant Triton tumor: a cytogenetic and molecular cytogenetic study. J Clin Pathol 57:1172-1178

78. Kresse SH, Skarn M, Ohnstad HO et al (2008) DNA copy number changes in high-grade malignant peripheral nerve sheath tumors by array CGH. Mol Cancer 7:48

79. Storlazzi CT, Brekke HR, Mandahl N et al (2006) Identification of a novel amplicon at distal $17 \mathrm{q}$ containing the BIRC5/survivin gene in malignant peripheral nerve sheath tumours. J Pathol 209:492-500

80. Schmidt H, Würl P, Taubert H et al (1999) Genomic imbalances in $7 \mathrm{p}$ and $17 \mathrm{q}$ in malignant peripheral nerve sheath tumors are clinically relevant. Genes Chromosomes Cancer 25:205-211

81. Skotheim RI, Kallioniemi A, Bjerkhagen B et al (2003) Topoisomerase-II $\alpha$ is upregulated in malignant peripheral nerve sheath tumors and associated with clinical outcome. J Clin Oncol 21:4586-4591

82. Schmidt H, Taubert H, Würl P et al (2001) Cytogenetic characterization of six malignant peripheral nerve sheath tumors: comparison of karyotyping and comparative genomic hybridization. Cancer Genet Cytogenet 128:14-23

83. Mantripragada KK, Spurlock G, Kluwe L et al (2008) Highresolution DNA copy number profiling of malignant peripheral nerve sheath tumors using targeted microarray-based comparative genomic hybridization. Clinical Cancer Res 14:1015-1024

84. Carroll SL, Stonecypher MS (2004) Tumor suppressor mutations and growth factor signalling in the pathogenesis of NF1associated peripheral nerve sheath tumors. I. The role of tumor suppressor mutations. J Neuropathol Exp Neurol 63:1115-1123

85. Przygodzki RM, Finkelstein SD, Keohavong P et al (1997) Sporadic and thorotrast-induced angiosarcomas of the liver manifest frequent and multiple point mutations in KRAS-2. Lab Invest 76:153-159

86. Naka N, Tomita Y, Nakanishi H et al (1997) Mutations of p53 tumor suppressor gene in angiosarcoma. Int J Cancer 71:952-955

87. Zietz C, Rossle M, Haas C et al (1998) MDM2 oncoprotein overexpression, p53 gene mutation, and VEGF upregulation in angiosarcomas. Am J Pathol 153:1425-1433

88. Domfeh AB, Fichera M, Hunt JL (2006) Allelic loss of 3 different tumor suppressor gene loci in benign and malignant endothelial tumors of the head and neck. Arch Pathol Lab Med 130:1184-1187

89. Ahmad SA, Patel SR, Ballo MT et al (2002) Extraosseous osteosarcoma: response to treatment and long-term outcome. J Clin Oncol 20:521-527

90. Jensen ML, Schumacher B, Jensen OM et al (1998) Extraskeletal osteosarcomas. A clinicopathologic study of 25 cases. Am J Surg Pathol 22:588-594
91. Mertens F, Larramendy M, Gustavsson A et al (2000) Radiationassociated sarcomas are characterized by complex karyotypes with frequent rearrangements of chromosome arm 3p. Cancer Genet Cytogenet 116:89-96

92. Mohamed AN, Zalupski MM, Ryan JR et al (1997) Cytogenetic aberrations and DNA ploidy in soft tissue sarcoma. A southwest oncology group study. Cancer Genet Cytogenet 99:45-53

93. Lau CC, Harris CP, Lu XY et al (2004) Frequent amplification and rearrangement of chromosomal bands 6p12-p21 and 17p11.2 in osteosarcoma. Genes Chromosomes Cancer 39:11-21

94. Selvarajah S, Yoshimoto M, Ludkovski O et al (2008) Genomic signatures of chromosomal instability and osteosarcoma progression detected by high resolution array CGH and interphase FISH. Cytogenet Genome Res 122:5-15

95. Bayani J, Zielenska M, Pandita A et al (2003) Spectral karyotyping identifies recurrent complex rearrangements of chromosomes 8, 17, and 20 in osteosarcomas. Genes Chromosomes Cancer 36:7-16

96. Bridge JA, Nelson M, McComb E et al (1997) Cytogenetic findings in 73 osteosarcoma specimens and a review of the literature. Cancer Genet Cytogenet 95:74-87

97. Sandberg AA, Bridge JA (2003) Updates on the cytogenetics and molecular genetics of bone and soft tissue tumors. Osteosarcoma and related tumors. Cancer Genet Cytogenet $145: 1-30$

98. Montgomery EA, Devaney KO, Giordano TJ et al (1998) Inflammatory myxohyaline tumor of distal extremities with virocyte or Reed-Sternberg-like cells: a distinctive lesion with features simulating inflammatory conditions, Hodgkin's disease, and various sarcomas. Mod Pathol 11:384-391

99. Meis-Kindblom JM, Kindblom LG (1998) Acral myxoinflammatory fibroblastic sarcoma: a low-grade tumor of the hands and feet. Am J Surg Pathol 22:911-924

100. Lambert I, Debiec-Rychter M, Guelinckx P et al (2001) Acral myxoinflammatory fibroblastic sarcoma with unique clonal chromosomal changes. Virchows Arch 438:509-512

101. Baumhoer D, Glatz K, Schulten H-J et al (2007) Myxoinflammatory fibroblastic sarcoma: investigations by comparative genomic hybridization of two cases and review of the literature. Virchows Arch 451:923-928

102. Mansoor A, Fidda N, Himoe E et al (2004) Myxoinflammatory fibroblastic sarcoma with complex supernumerary ring chromosomes composed of chromosome 3 segments. Cancer Genet Cytogenet 152:61-65

103. Hallor KH, Sciot R, Staaf J et al (2009) Two genetic pathways, $t$ $(1 ; 10)$ and amplification of $3 \mathrm{p} 11-12$, in myxoinflammatory fibroblastic sarcoma, haemosiderotic fibrolipomatous tumour, and morphologically similar lesions. J Pathol 217:716-727

104. Ida CM, Rolig KA, Hulshizer RL et al (2007) Myxoinflammatory fibroblastic sarcoma showing $\mathrm{t}(2 ; 6)(\mathrm{q} 31 ; \mathrm{p} 21.3)$ as a sole cytogenetic abnormality. Cancer Genet Cytogenet 177:139-142

105. Laskin WB, Silverman TA, Enzinger FM (1998) Postradiation soft tissue sarcomas. An analysis of 53 cases. Cancer 62:2330-2340

106. Wiklund TA, Blomqvist CP, Raty J et al (1991) Postirradiation sarcoma. Analysis of a nationwide cancer registry material. Cancer 68:524-531

107. Lagrange JL, Ramaioli A, Chateau MC et al (2000) Sarcoma after radiation therapy: retrospective multi institutional study of 80 histologically confirmed cases. Radiation therapist and pathologist groups of the Federation Nationale des Centres de Lutte Contre le Cancer. Radiology 216:197-205

108. Inoue YZ, Frassica FJ, Sim FH et al (2000) Clinicopathologic features and treatment of postirradiation sarcoma of bone and soft tissue. J Surg Oncol 75:42-50

109. Nakanishi H, Tomita Y, Myoui A et al (1998) Mutation of the p53 gene in postradiation sarcoma. Lab Invest 78:727-733 